NASA

Technical

Paper

3217

1992

Shortcomings in Ground

Testing, Environment

Simulations, and

Performance Predictions

for Space Applications

E. G. Stassinopoulos

Goddard Space Flight Center

Greenbelt, Maryland

G. J. Brucker

GE Astro-Space Division

West Long Branch, New Jersey 


\title{
SHORTCOMINGS IN GROUND TESTING, ENVIRONMENT SIMULATIONS, AND PERFORMANCE PREDICTIONS FOR SPACE APPLICATIONS
}

\author{
E.G. Stassinopoulos \\ NASA-Goddard Space Flight Center \\ Greenbelt, MD \\ and \\ G.J. Brucker \\ GE Astro-Space Division \\ West Long Branch, NJ
}

\begin{abstract}
This document addresses the issues involved in radiation testing of devices and subsystems to obtain the data that are required to predict the performance and survivability of satellite systems for extended missions in space. The problems associated with space environmental simulations, or the lack thereof, in experiments intended to produce information to describe the degradation and behavior of parts and systems are discussed. Several types of radiation effects in semiconductor components are presented, as for example: ionization dose effects, heavy ionand proton-induced Single-Event-Upsets (SEUs), and SingleEvent-Transient-Upsets (SETUs). Examples and illustrations of data relating to these ground testing issues are provided. The primary objective of this presentation is to alert the reader to the shortcomings, pitfalls, variabilities, and uncertainties in acquiring information to logically design electronic subsystems for use in satellites or space stations with long mission lifetimes, and to point out the weaknesses and deficiencies in the methods and procedures by which that information is obtained.
\end{abstract}

\section{Introduction}

The requirements of testing parts for space applications has progressed over the years from simple to complex problems and requirements. The increase in complexity has been driven by advances in technology and a better understanding of environmental threats and their characterization.

There are a host of components affected by radiation in space: the spacecraft structure itself and the materials used on the exterior of the structure such as thermal control coatings, cables and their insulation, antenna materials with thermal and discharge control coatings, solar arrays with their special coatings, and sensors of various types. The degradation of these components plus the electronic subsystems within boxes located inside the spacecraft structure, or even sometimes outside it, may be in the form of a slow, gradual decay.

Also, over the years, mission lifetimes have continuously increased from 6 months (e.g., TIROS Weather Satellite) to 10 years and more for communications spacecraft, and now for the Space Station Freedom (presently in planning and development stages) to a requirement of 30 years in a low-altitude Earth orbit.

Design specifications for these components must address the structural integrity (e.g., fatigue, brittling and powdering), mo- lecular/chemical breaking of bonds, and the changes in electrical and magnetic properties. Parameters of semiconductor devices (for example: leakage current, speed, threshold voltage and gain) degrade to a level where functional failure of the parts, and the subsystems they control, occurs. In some instances, these failures can lead to the loss of the satellite or impair the complete achievement of mission objectives.

Before the radiation effects in electronics can be addressed, definitions of these effects have to be formulated. Environments and their particle types, spectral distributions, interaction modes and rates, and potential kinds of interactions involving the production of secondary or tertiary particles must be defined.

\section{Shortcomings in Ground Testing}

Design engineers must have the necessary data that characterize the degradation profile of microclectronic components for the spacecraft and orbits. Ground tests introduce a number of shortcomings due to the constraints of time, cost, and availability of facilities. The radiation facilities are usually restricted to discrete single sources, monoenergetic at any instant of time, with a unidirectional and narrow beam, and high rates of exposure. In contrast to these conditions, the radiation in space consists of a simultaneous incidence of all particle species populating a given region, all energies (complete spectral distributions), low rates arriving instantaneously from all directions (i.e., omnidirectional), and mostly isotropic. Consequently, ground test simulations introduce a non-equivalence and a lack of synergism which may lead to poor or totally wrong designs unless corrective actions are applied to compensate for these shortcomings. The important thing to remember is that electrons, protons, cosmic rays, solar flare particles (if applicable), and ultraviolet sources of radiation are simultaneously incident on a satellite.

In some ground tests, the experimenters have designed exposure fixtures that move during the irradiation in such a manner so as to eventually expose all sides of a target to the beam, trying to produce an equivalent omnidirectional incidence. Of course, this does not simulate the simultaneous arrival of radiation on the target in space; that is, from all directions instantaneously. Most of the modern electron, proton, or heavy ion facilities can achieve relatively low rates, but for controlled beams, the limiting value is determined by the loss of stability of the beam. For beams attenuated by degraders, the limiting values are deter- 
mined by the purity or integrity of the beam. In the case of ground testing with Co-60 gammas, the rates are usually high; Co-60, cesium, or other low-intensity radioactive sources also have been used in some low dose-rate testing. However, this type of radiation does not exist in space.

Most of the ionization dose data have been taken at high rates. Such accelerated tests make it necessary to look for the post-irradiation effects (PIE) that take place in MOS devices, such as annealing, reverse anneal ing or rebound, gain losses, and speed degradation.

These effects may cause a part that passes an accelerated test to fail during the period following the exposure, or the reverse can occur, that is, a part that fails an accelerated test can pass a PIE test (e.g., leakage current).

Operating temperatures in spacecraft electronic boxes may be higher and varying in values compared to an experiment conducted at room temperature. This forces the designer to extrapolate the data from the experimental value to the operating temperature in space.

A special requirement for most of the heavy ion test facilities (SEU experiments) is that devices-under-test (DUTs) be delidded since the range of the ions is not sufficient to penetrate the lids. Facilities with high-energy beam ions, in the $\mathrm{BeV}$ range, permit devices to be exposed with their lids on. Of course, in space, devices are lidded; therefore delidded data do not represent a real condition and hence are suspect as to their accuracy and validity. However, there are also other issues conceming high-energy versus low-energy ion beams which will be discussed elsewhere in this paper. Another issue is the restriction of low-energy ion beams to angles of incidence in the range of $0^{\circ}$ to $70^{\circ}$, whereas in space, particles arrive from all $4 \pi$ directions. Uncertainties duc to irradiation at angles other than $0^{\circ} \mathrm{can}$ impact the determination of experimental upset cross sections. This is because the cross section is calculated by dividing the number of errors by the particle fluence multiplied by the cosine of the incident angle $\boldsymbol{\theta}$, where the latter is calculated relative to the normal to the device. This adjustment is only correct when the cross-sectional area of the device's sensitive volume is large $(900$ square micrometers). In the case of a small surface area, the correct adjustment factor will lie between the values of $\cos ^{2} \theta$ and $\left[\cos \theta+\cos ^{2} \theta\right]$ (1). In all of the above shortcomings, there remains the issue of discrete particles and energies used for independent tests versus the possible synergistic effects in the space environment.

The community must accept the fact that ground testing is both insufficient and inadequate to duplicate correctly the actual space radiation environment. Radiation researchers must compensate and account for the many serious deficiencies by trying to reconcile the simplistic and primitive laboratory test results with the complex, free-space reality.

\section{Radiation Units}

In order to standardize ground test data, the radiation community has adopted the units of "rad" or "grey" (= 100 rads) for calculating ionization doses. The rad is defined as the deposition of $100 \mathrm{ergs} \mathrm{of} \mathrm{energy} \mathrm{for} \mathrm{a} \mathrm{gram} \mathrm{of} \mathrm{target} \mathrm{material.} \mathrm{Its} \mathrm{primary} \mathrm{use}$ is in connection with materials and electronic devices for quan- tifying the damage and degradation effects. There have been a great number of studies and experiments in the past several years to understand the drawbacks in the use of this unit to quantify radiation effects. The experimental results have demonstrated that the rad unit does not al ways account for the non-equivalence of sources, energies, and rates. There is a need for defining a new unit that could correct this deficiency, possibly a unit similar to the Radiation Biological Effectiveness (RBE) or Quality Factor (QF) in radiobiology.

\section{Testing Objectives}

The primary objectives of ground tests are to identify and understand the physical processes of interactions in devices, and to evaluate performance of parts fabricated with different technologies and by different production methods. The results of achieving these objectives then lead to the additional objectives of establishing whether components will perform to specifications in the natural or man-made environment and, subsequently, developing survivable, functional, and healthy systems for use in space.

\section{Specific Testing Concerns}

In order to achieve the above goals, the researchers must design the tests with careful attention to an extensive list of concerns. In the Single Event Upset (SEU) tests, the Linear Energy Transfer (LET) threshold and the asymptotic crosssection may be modified by the devices under test (DUTs) accumulating too much ionization dose, the occurrence of multiple errors during the measurements, or imprint effects. The range of heavy ions relative to device dimensions, package shadowing at large angles, and inequality of LET due to ion track distributions (which depend on energy and specie) are some of the SEU test concerns. The experimental design must take these concerns into account or the data may turn out to be unacceptable. Tests must be conducted for temperatures that are expected in the satellite.

There is also a list of concerns relative to ionization dose tests. In testing MOS or CMOS parts, dose-rate effects, as for example: recovery, rebound, and latent functional failure (e.g., due to post-rad speed degradation), must be taken into account. Bias and temperature dependencies, and static versus dynamic operation during exposure are also concerns in this type of testing.

In both SEU and ionization experiments, adequate sample size (mavericks distort data) and a sufficient number of runs per sample are of concern to the experimenter.

\section{Trends in Testing}

Trends in testing parts evolve with time in response to pressures, needs, recognition by program managers of their importance, and of course, to parts becoming available. These approaches, methods, and procedures are constantly being refined, upgraded, and improved as new insight is obtained, knowledge accumulates, and requirements become more demanding. 
These "single part/single run" tests evolve into "single part/ many runs," "many parts/single run," and finally "many parts/ many runs." Similarly, "one source/one energy" evolves to "one source/many energies," "several sources/one energy," and finally, "several sources/many energies."

The increase in the density of transistors has also established a trend from discrete parts testing to Small-Scale Integration (SSI), to Large-Scale Integration (LSI), and finally, to Very Large Scale Integration (VLSI) and Very High Scale Integrated Circuit (VHSIC).

The evolution or advance in the state-of-the-art in basic technology has also set a trend from testing bulk bipolar to bulk CMOS, and at the present time, CMOS on sapphire and CMOS on insulator.

\section{Factors-Variabilities-Beam Concerns Affecting Test Results}

\section{A. Device Dependent}

The ionization dose hardness of CMOS parts is affected by the types of gates (aluminum versus silicon), by transistor type (n-versus p-channel), oxide, and circuit design. The most significant of these factors is the gate oxide, where the thickness dominates the hardness capability of an oxide, and to a lesser extent, the type of oxide, i.e., wet versus dry. Also, oxide growth temperature and subsequent process steps must be maintained equal to or less than the growth temperature in order to harden the oxide. The hardness varies as the square of the oxide thickness for positive oxide-trapped charge, although for some oxides individual researchers have claimed that it varies with the cube of the thickness. The magnitude of negative charge traps at the silicon dioxide-silicon interface is dependent on whether the oxide is wet or dry, and particularly on its hydrogen content or other impurities.

Feature size is the spacing between the source and drain of a CMOS part. This dimension is critical to establishing the SEU sensitivity of a device. The smaller the dimension is, the greater the SEU sensitivity of a device. Finally, the organization of a part, for example, whether it be a $64 \mathrm{Kx} 1$ or an $8 \mathrm{Kx} 8$, will modify and impact the SEU sensitivity of memory systems.

\section{B. Facility Dependent}

Radiation sources and energies are important factors in parts testing. In particular, for CMOS devices, the ionization damage induced by different types of sources and energies may not be equivalent to each other when expressed in units of rads (Si) or greys. Some illustration of this fact will be presented later in this paper. For those tests where a particle generator is utilized, there are beam factors that are important, such as uniformity of intensity, spread of energy, and contamination of the beam by unwanted particles. This latter concern is particularly significant in SEU tests. Of course, dosimetry must be good for either ionization dose from radioactive sources or from particle beams, and for SEU heavy ion tests, particle counting, specie identification, and LET measurement are important.

\section{Experiment Dependent}

Factors affecting test results that are dependent on the experiment are the ionization dose, dose rate, bias, time of measurement, operating mode, fluence, flux, LET, and beam current. Some of these are common to both particle and ionization dose types of experiments (for example, bias and temperature). The significance and impact of any factor on test results is dependent on circuit design.

\section{Variabilities-Uncertainties}

Test data are impacted by variations from run to run for the same sample, part to part from the same lot, lot to lot for the same vendor, and vendor to vendor for the same process. The uncertainty in measurement from run to run is controlled by the capability of the facility, whereas the reproducibility of parts from the same lot, or lot to lot from the same vendor is strongly dependent on the vendor's control of his process. Ionization damage or upset response is too dependent on process variables to show small variations from vendor to vendor for the same part type. A designer could not infer the damage properties of the same part from one vendor on the basis of measurements made with another vendor's part. This is more likely to be possible for tests to evaluate displacement damage in silicon due to neutrons or protons.

\section{E. Beam Concerns}

Beam impurities or contaminants can distort data and invalidate results. The capability of the generator facility to ensure the purity of the beam determines the confidence the designer may have in the test results. Non-uniformity over the total crosssectional area from valleys or hot spots may produce wrong and misleading results. A single, well-defined peak in the energy distribution is particularly necessary in SEU testing so as to have a well-defined LET for the heavy ions. Since the heavy ion or proton exposures may take minutes, the stability of the beam is also important. The energy and particle specie for SEU tests determine the range of the particle, and thus, its ability to penetrate the sensitive volume of the device under test (DUT). Invalid data can be obtained if the range of the beam particle is not adequate to penetrate the device.

\section{Concerns of Shielding Calculations}

\section{A. Variables Directly Affecting Dose Evaluation}

The primary environment incident on a spacecraft must be defined by specie and spectral distribution, as must be the secondaries produced within the spacecraft. With these inputs and with descriptions of shield geometry, shield composition, and a shield evaluation program, a shielding analysis can be carried out. The dose points and target composition allow dose evaluation for specific devices in boxes and within their packages to be accomplished. To assess the applicability and accuracy of such an analysis, the assumptions and approximations should be provided in the documentation. 


\section{B. Functional Dependence of Shielding Calculations}

The dependence of dose at a location in a spacecraft is a very complex function of about 18 variables (2). It can be described mathematically by a functional expression in which the dose $D$ is given by:

$$
D\left(S, S, E, e, Z, z, T, G, I, H, Y, l, M, \Delta, A, C, X_{l} X_{2}\right)
$$

where the definitions of the variables are as follows:

$S:$ primary radiation source which may contain protons, electrons, ctc.

$s:$ secondary radiation which may be bremsstrahlung, neutrons, etc.

E: energy of incident radiation given as a spectral distribution. $e:$ energy of secondary radiation as a spectral distribution.

$Z$ : atomic number of shield material, e.g., aluminum, iron, tantalum, etc.

z: atomic number of target material, e.g. silicon for a device, water for tissue, etc.

$T$ : areal thickness of shield in $\mathrm{gm} / \mathrm{cm}^{2}$.

$G$ : shicld gcometry, e.g., finite slab, solid sphere, spherical shell, cylinder, etc.

I: orbit inclination, which may vary from 0 to 90 degrees.

$H$ : orbit altitude, e.g., $300 \mathrm{~km}$ for LEO, $35,790 \mathrm{~km}$ for GEO.

$Y$ : mission epoch, which is related to the launch date of the spacecraft.

$t$ : mission duration, which determines the accumulated dose and in conjunction with $Y$ is used to select solar maximum or minimum models to be used in the calculation.

$M$ : magnetic field: involves the selection of a particular magnetic field appropriate for that mission and its objectives.

$\Delta$ : ficld extrapolation: involves the secular variation of a given field model.

A: calculational approach: describes the complexity of the method employed; e.g., 2-dimensional, 3-dimensional, simple, complex, etc.

$C$ : transport code, e.g., SHIELDOSE, TIGER, NOVICE, etc.

$X_{1}$ : orbit eccentricity for elliptical orbits.

$X_{2}:$ any other parameters required to complete the functional dependence of dose, $D$.

A flowchart summarizing these variables and applications is given in Figure 1.

\section{Ionization Dose Experiments}

\section{A. Non-Equivalence of Sources and Energies}

Ionization damage in MOS transistors depends on the type of radiation source and particle energy. In space, electrons and protons generate ionization charge by producing electron-hole pairs. The number of these is dependent on the stopping power of the particles, and in the case of Co-60 gammas, it is the Compton and photoelectrons that are produced by the gamma-ray interactions in the device material. In the MOS device, it is the $\mathrm{SiO}^{2}$

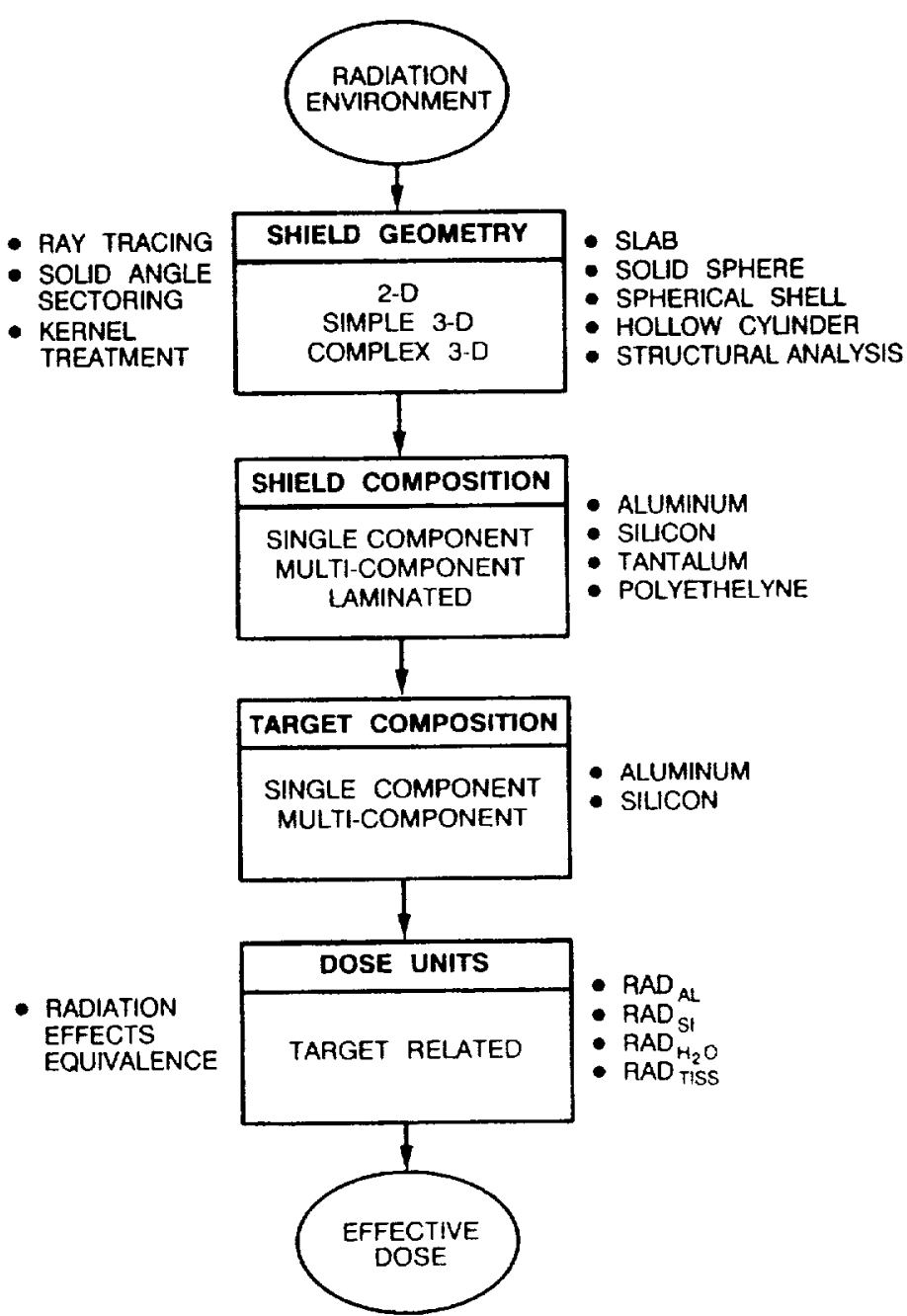

Figure 1. Shielding Analysis Flow Chart.

gate and field oxide materials that are the primary chargetrapping regions, and consequently are responsible for damage effects. However, a dose in units of rads (or grey), which is a measure of energy deposited in these oxides, does not take into account the differences in the rates of production and effective amounts of charge available for trapping in the oxides. Thus, time-dependent charge transport and annealing mechanisms produce different values of threshold voltage shifts in $n$-channel transistors. This effect is illustrated by the curves in Figure 2, which show the non-equivalent damage sensitivity (or voltage shift per dose), $\Delta \mathrm{V}_{\mathrm{TN}} / \mathrm{DOSE}\left[\mathrm{volts} / \mathrm{Krad}\left(\mathrm{SiO}^{2}\right)\right]$, as a function of particle energy and type compared to Cobalt- 60 gammas, for a CMOS/Bulk, CD4007 soft part (3). There are three curves in this figure, one that describes the damage incurred by irradiating the devices with Co-60, which is illustrated by a band or range of voltage shifts horizontal to the $\mathrm{x}$-axis, a separate curve for electrons, and one for protons. The standard deviations of the data are indicated by error bars on each data point for the two particles, and by the width of the band representing the Co- 60 gamma damage. The non-equivalence in damage for the same rad dose is clearly shown for these three different radiation sources. 


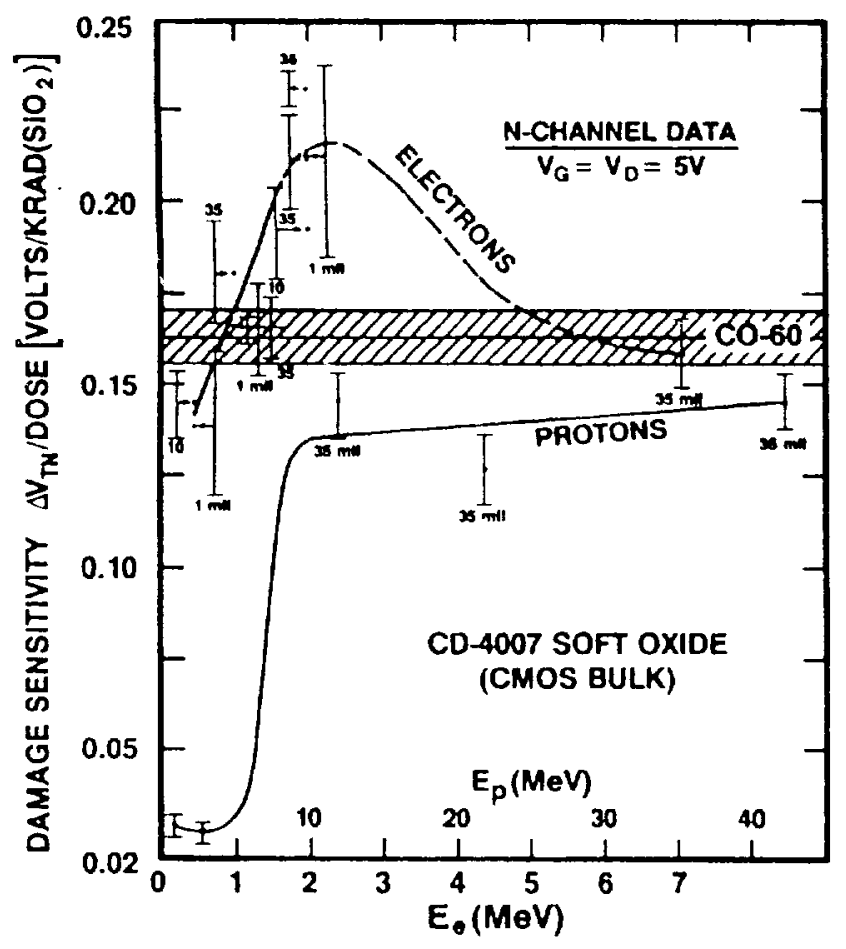

Figure 2. Dependence of Damage Sensitivity $\left(\Delta V_{T} \sqrt{D O S E}\right)$ on Radiation Type and Energy: Non-Equivalence.

\section{B. Non-Equivalence of Annealing/Rebound}

It was pointed out in the previous section that annealing properties impact post-rad damage states in $\mathrm{n}$-channel transistors. Figure 3 demonstrates the non-equivalence of annealing for devices irradiated by gamma rays, electrons, and protons (4). The plots of percentage recovery of threshold voltage versus annealing times show that there are distinct differences in the recovery properties of electron-induced damage compared to protons for these soft parts, whereas the samples irradiated with Co-60 gammas anneal as a function of time in close agreement with samples irradiated with 1-MeV electrons. Since the space environment is dominated by trapped electron spectral distributions, it would produce damaged devices, which would tend to follow the curves for the combined electron sources of .5-, 1-, and 2 $\mathrm{MeV}$ electrons. The annealing properties of $\mathrm{Co}-60$ ground-tested parts would not predict the space behavior.

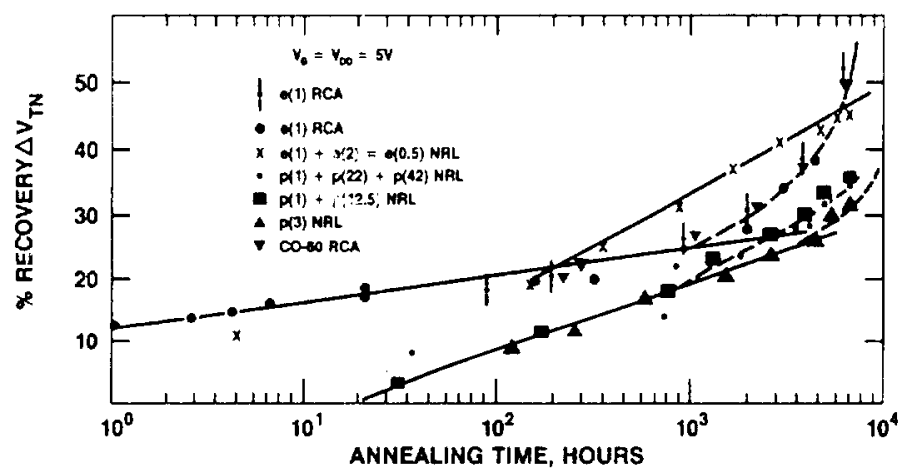

Figure 3. Non-Equivalence of Annealing for Devices Irradiated with Electrons, Protons, and Co-60 Gamma Rays.
The same conclusion also applies to the parts irradiated by combinations of 1-, 3-, 12-, and 42-MeV protons; namely, Co-60 test data do not predict the behavior of parts irradiated by a trapped proton spectrum in space. In contrast to the electrons, the proton annealing data indicate that a source of one energy, 3 $\mathrm{MeV}$, or a combination of two or three energies, caused samples to follow similar annealing curves. Note that there are two distinct annealing time constants for samples irradiated by the electron spectrum. There also seems to be a similar trend of a second time constant of recovery for the proton-irradiated samples at about $5000-10000$ hours post-irradiation. Of special interest is also the approximately 24-hour delay in the appearance of a measurable annealing shift in these parts. No explanation is yet available for this delayed response.

Figure 4 shows the case for a bias condition in a CMOS inverter of the gate voltage equal to zero, $V_{8}=0$, and the drain voltage equal to $5 \mathrm{~V}, \mathrm{~V}_{\mathrm{D}}=5$, and for irradiations with single, discrete, monoenergetic electrons, protons, and Co-60 gamma rays (5). Figure 4 contains plots of percentage recovery of $\Delta \mathrm{V}_{\mathrm{TN}}$ versus annealing time. In agreement with the previous annealing data in Figure 3, there is close correlation in the $1-\mathrm{MeV}$ and 7$\mathrm{MeV}$ electron curves and the Co-60 curves. However, for all the other electron, proton, and alpha particle curves, Co-60 test re-

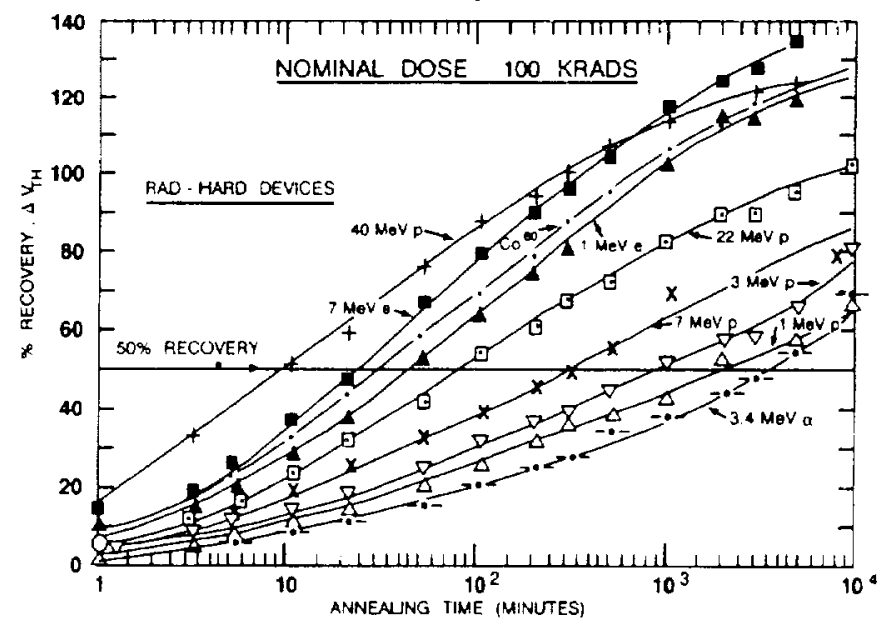

Figure 4. Percentage Recovery vs. Time for N-Channel Data $\left(\mathrm{V}_{\mathrm{B}}=0, \mathrm{~V}_{\mathrm{D}}=5\right)$.

sults would not come close to predicting the space particle data. If one were to measure the $50 \%$ annealing of $\Delta \mathrm{V}_{\mathrm{TN}}$ for a sample irradiated by an unknown source, then the data indicate that one could differentiate and identify the unknown source and energy on the basis of this parameter, provided the ionization dose imparted to the devices was on the order of $100 \mathrm{Krads}$. These samples were all irradiated by that same dose but the effective trapped charge is not the same for all these sources, particularly for the lower energy protons and alpha particles where the higher stopping powers of these particles initially produce greater charge densities. The recombination losses for these low-energy particles aregreater and thus the effective trapped charge is greater for the higher energy particles with lower stopping power. The threshold shift damage is greater and the recovery from damage is faster for the Co-60 and higher energy protons or the 1- to 2- MeV electron-irradiated devices. 


\section{Time of Measurement}

Time of measurement was identified in a previous section as a ground test concem. The reason for this claim is illustrated in Figure 5 by the in-situ measurements of threshold shifts for $n$ - and p-channel transistors for the two standard CMOS inverter bias conditions and for a Co-60 source (6). The $\mathrm{n}$-channel transistor with a gate bias of zero volts starts to recover during the exposure so that if measurements of damage are made at the termination of the irradiation, at a dose of $100 \mathrm{Krads}$, the measured damage at that time would be: 1) less than that for the 5-volt gate bias, and 2) less than at lower dose levels during that exposure. In contrast, the dose requiring only about 6 minutes of irradiation time would indicate that the damage is the same for either bias under these test conditions (source and fixed dose rate). This effect could cause device failure at a low dose without being detected in a high-dose experiment. Thus, the time at which the measurement is made relative to the source's dose level can lead to different conclusions concerning a device's worst-case bias condition for damage.

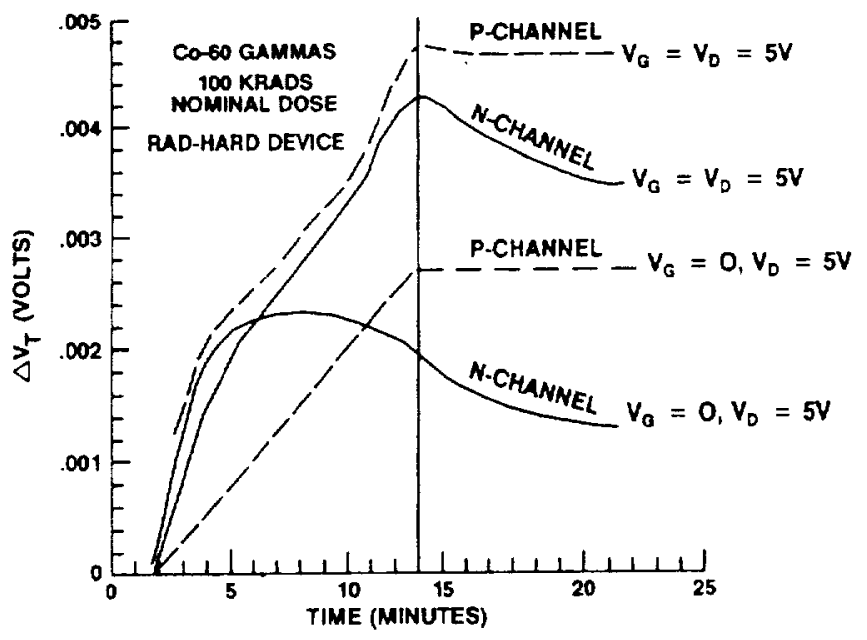

Figure 5. Threshold Voltage Shift vs. Time During and After Irradiation: Time of Measurement Dependence.

\section{Rate Effect}

Experimental data for the dependence of damage on sources were presented up to this point. But similarly important properties are associated with rate effects $(7,8)$. This is demonstrated for a CMOS/SOS CDP1821, 1K static memory in Table 1 (7) for dose rates ranging from 5.9 rads/hour to $530 \mathrm{krads} /$ hour, covering change in dose rates by a factor of about 100 . It can be seen that the failure dose measured for these parts at a high dose rate increased significantly at the lower dose rates. At an extrapolated space dose rate of about $.5 \mathrm{rad} /$ hour, expected behind a shield of about $1 \mathrm{gm} / \mathrm{cm}^{2}$ aluminum for a worst-case pass through the South Atlantic Anomaly, the failure level has risen by a factor of 3 to 4 .

\section{E. Bias Dependence}

Another shortcoming of ground testing is illustrated by the field and angular dependence data for the Co-60 and low-energy proton sources in Figure 6 (3). A gamma-cell 220, Cobalt 60 source contains pencils of cobalt arranged around a cylindrical exposure volume so there is no specific gamma-ray direction. The angular dependence for the lower energy protons and for the $1-\mathrm{MeV}$ electrons is not simulated by Co-60, nor is the field dependence. The damage dependence on the field indicates that the least damaging operating voltage is about 5 volts for these parts.

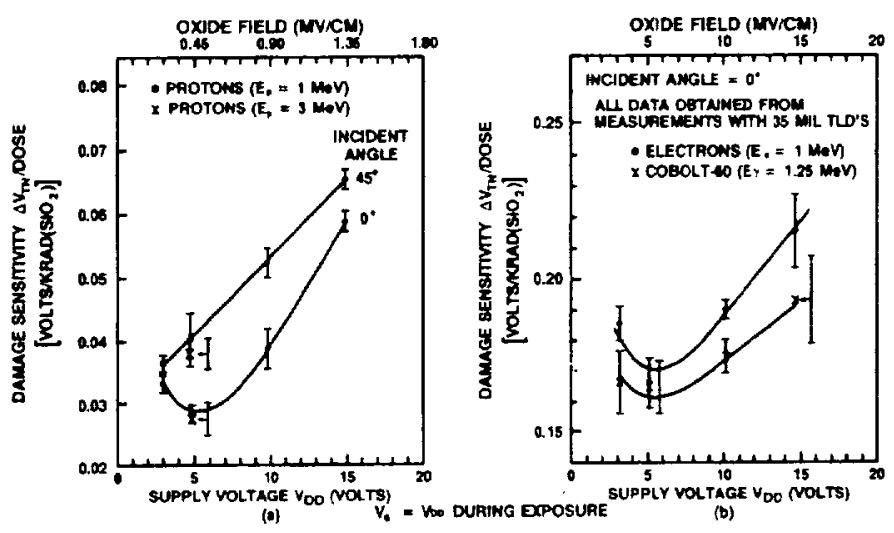

Figure 6. Dependence of Damage Sensitivity $\left(\Delta \mathrm{V}_{\mathrm{T}} / \mathrm{DOSE}\right)$ on Voltage of Oxide Field and Incident Angle of Radiation Beam.

\section{SEU Concerns}

\section{A. Trends in Device Evolution}

The state of the art in integrated circuits (ICs) is continually advancing with new technologies, smaller feature sizes, and higher levels of integration. The newer technologies with smaller feature sizes, such as CMOS fabricated on sapphire (CMOS/ SOS) and CMOS on insulator (CMOS/SOI) with feature sizes (source-drain spacing) of 1.25 microns, have been hardened against SEU by introducing additional nodal capacitance in the 6-transistor memory cell in memory devices (e.g., CMOS/SOS, $65 \mathrm{~K} \mathrm{RAM}$ ). In the case of a $64 \mathrm{~K} \mathrm{CMOS/SOI} \mathrm{RAM,} \mathrm{the} \mathrm{harden-}$ ing technique has been to introduce cross-coupled resistors in the feedback loop within the cross-coupled inverters of the memory cells. Basically, it has been the shrinking of feature sizes that has caused an increase of sensitivity to SEU. The smaller feature size causes the critical charge generated by a heavy ion hit to decrease. It has been shown experimentally (9) that the critical charge for upset varies as the square of the feature sizc, and that this dependence is the same for various technologies such as bipolar, CMOS/Bulk, NMOS/Bulk, and CMOS/SOS. Both of these hardening techniques, the increased nodal capacity and the cross-coupled resistors, work very well and can harden a part (even with a 1.25-micron feature size) to a very high level of immunity. Error rates for RAMs of either technology on the order of $10^{-11}$ to $10^{-12}$ errors/bit-day for a $90 \%$ worst-case environment in a geostationary orbit are possible. The capacitive approach is 
easier to process, and does not affect the part's speed but it increases the area of the cells. In contrast, the resistor approach is more difficult to process, slows down the write speed of the part, and does not impact the area of the cell, but it now has a limited temperature range of operation. This temperature effect is due to the temperature dependence of the resistors. At too low a temperature, the resistor value increases so that the speed of the part is significantly reduced. For too high a temperature, the resistance decreases so that the SEU hardness decreases significantly. Of course, the capacitor is not sensitive to temperature so that the temperature range of operation can be $-55^{\circ} \mathrm{C}$ to $125^{\circ} \mathrm{C}$.

\section{B. Objectives of SEU Experiments}

In order to properly address the SEU problems in a spacecraft system, one requires the basic SEU parameters of all the components. There is the JPL and AEROSPACE data bank that may be searched for this information; however, in many cases tests have to be performed to obtain the necessary data. The objectives of SEU experiments are to determine device sensitivity to soft errors and latchup, and to investigate the dependence of device performance on important variables and conditions such as temperature, bias, pattern sensitivity, and static versus dynamic operating modes. The ultimate objective is to obtain the corresponding standard SEU parameters of asymptotic cross section and threshold LET which can then be used to calculate and predict upset rates in space by folding in the expected mission-specific environment data.

\section{Causes of Change in SEU Sensitivity}

There are radiation effects occurring during the course of a SEU experiment or during a spacecraft's mission that alter the SEU sensitivity of parts. Accumulation of ionization dose in an experiment or in space will imprint the stored pattern in a RAM. Depending on operating conditions, bias, temperature (either during the exposure or after it), and also dose rate can cause SEU sensitivities of a RAM to increase or decrease.

Although a heavy-ion experiment does not normally expose test samples to high doses, a proton upset test may do exactly this. Any type of hardened RAM will require large fluences of protons in order to produce enough upsets so as to make cross-section measurements possible. For example, CMOS/SOS RAMs would require fluences equivalent to doses in the hundreds of Krads.

\section{LET Inequality}

When the heavy-ion environment in space was initially recognized as a threat, heavy-ion testing commenced in earnest and the LET parameter was used extensively to characterize the sensitivity of parts to SEU. Experimentally, researchers used angles of incidence ranging from $0^{\circ}$ to $70^{\circ}$ to effectively increase the normally incident LET by multiplying it with the secant of the incident angle, $\boldsymbol{\theta}_{i}$. This allowed a curve of error cross-section to be developed easily by the use of only one ion specie in some cases. However, as the testing became more sophisticated and knowledge accumulated, experimental results $(10,11,12)$ showed that the use of an equivalent LET given by "LET $x$ secant $\Theta_{i}$ " was only valid in some cases. In addition, the ionization charge in the distribution around an ion's track in silicon may influence the experimental SEU sensitivity of a device. Figures 7,8 , and 9 illustrate the reasons for this effect whereby track structure may alter SEU sensitivity. Figure 7 contains a plot of LET versus ion energy for copper ions of $25 \mathrm{MeV}$ and $395 \mathrm{MeV}$.

\section{CONVERSION RATE OF DEPOSITED ENERGY FOR Si:}

\section{$3.6 \mathrm{eV} / \mathrm{e}-\mathrm{h}$}

\section{MAXIMUM DELTA RAY ENERGY FROM CU IONS: IN Si

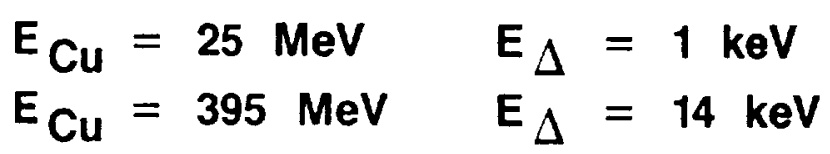

\section{STOPPING POWER OF Cu IN Si}

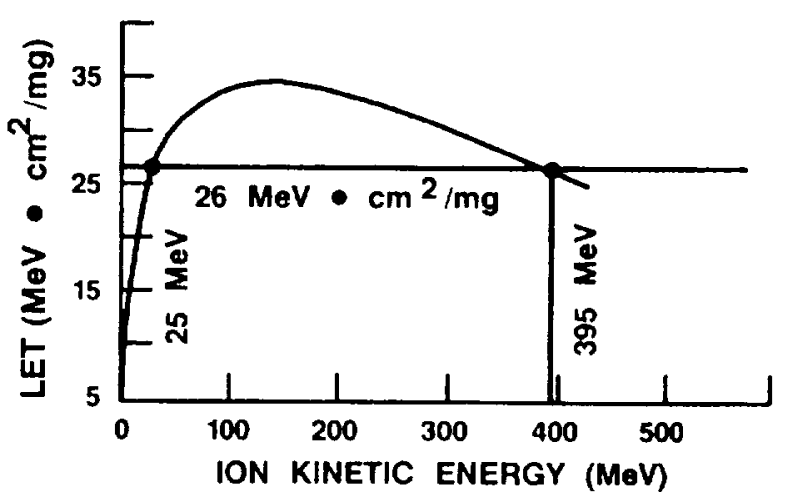

Figure 7. Inequality of LET Effects.

For these two energies, the stopping power (or LET) is the same, that is, slightly more than $25 \mathrm{MeV}-\mathrm{cm}^{2} / \mathrm{mg}$. However, the deltaray energies resulting from the passage through silicon of these two ions with vastly different energies are significantly different. It can be seen that the delta rays are $1 \mathrm{KeV}$ for the 25-MeV copper ion and $14 \mathrm{keV}$ for the $395-\mathrm{MeV}$ ion. Thus, the number of electron-hole pairs produced by these two delta rays differs by a factor of 14, and the distribution of charge around the tracks will be significantly different. This has been shown experimentally to produce differences in SEU sensitivity. Figure 8 (12) illustrates a plot of the charge concentration and its radial distribution around the central $\mathrm{Si}$ ion track as the ion enters the silicon target at zero micrometers and leaves the target at 20 micrometers. The $\mathrm{Si}$ ion energy is $186 \mathrm{MeV}$ and its LET is $8.02 \mathrm{MeV}-\mathrm{cm}^{2} / \mathrm{mg}$. Normal doping densities in silicon devices are about $10^{15}$ to $10^{16}$ atoms $/ \mathrm{cm}^{3}$. It can be seen that a density of electron-hole pairs 


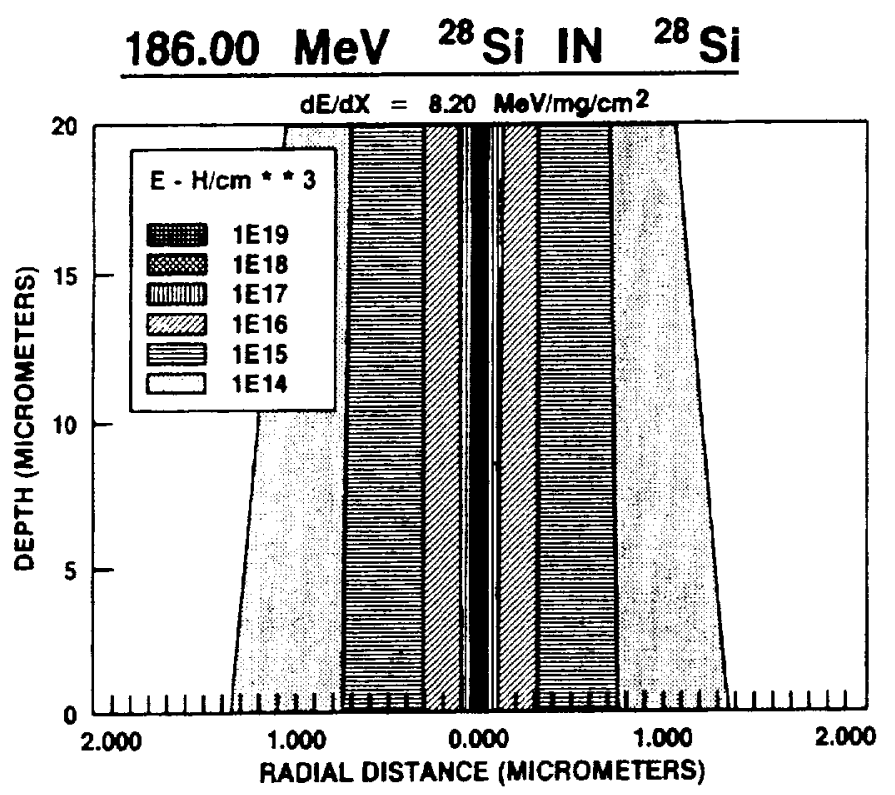

Figure 8. Charge Distribution for $186-\mathrm{MeV} \mathrm{Si}$ Ion in $\mathrm{Si}$ as a Function of Radial Distance from Center of Ion Track.

equal to the ambicnt, electron-holes $/ \mathrm{cm}^{3}$, impurity density occurs at a radial distance of .1 to $.7 \mu \mathrm{m}$ from the central track. In contrast to this situation, Figure 9 (12) shows a similar piot for $350-\mathrm{MeV}$ gold ions with a LET $=82.12 \mathrm{MeV}-\mathrm{cm}^{2} / \mathrm{mg}$. This LET is 10 times as large as the $\mathrm{Si}$ ions and this higher ionization power produces the very high density of $10^{19}$ electron-holes $/ \mathrm{cm}^{3}$ at the center of the ion path. This is wide and very dense, leading to a high recombination loss of charge relative to $\mathrm{Si}$ ions because of its wider distribution. Note that to reach the ambient device impurity region, the range of radial distance is now .18 to .225 micrometer. Experiments suggest that these tract properties can modify SEU sensitivities, thus, care has to be exercised in selecting ions to characterize the error cross-section of parts (10, $11,12)$.

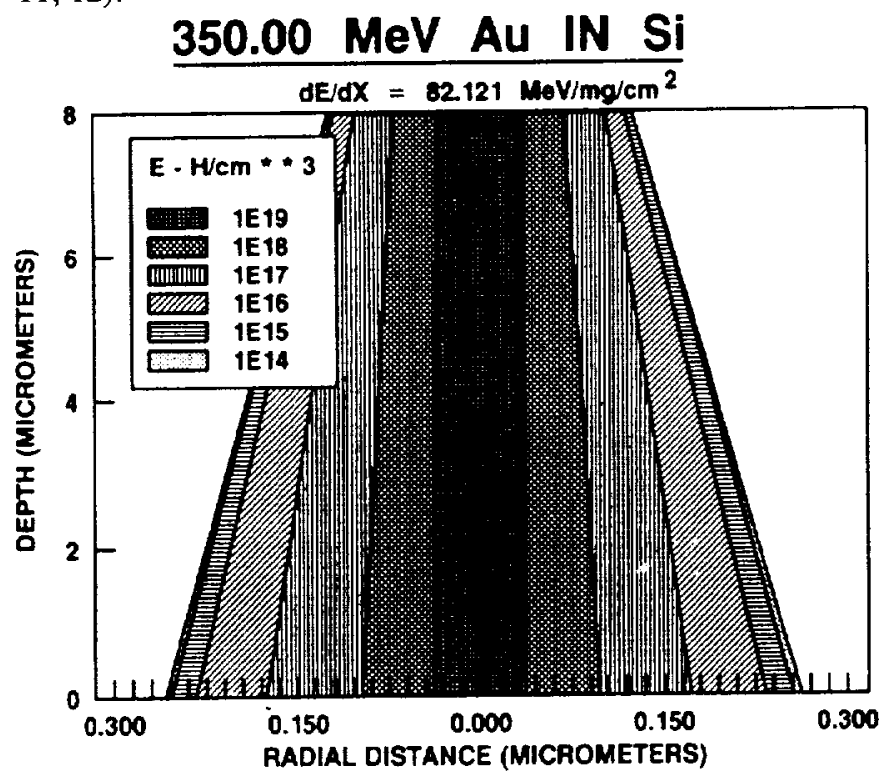

Figure 9. Charge Distribution for $350-\mathrm{MeV}$ Au Ion in $\mathrm{Si}$ as a Function of Radial Distance from Center of Ion Track.

\section{E. Imprint Effect}

The accumulation of ionization dose by a memory device will imprint a pattern stored in a memory cell. The reason for this effect is that the storage of a "one state" in a 6-transistor, crosscoupled inverter cell will turn on a pair of $n$ - and p-channel transistors, leaving the other pair in an off state. Thus, a pair of on-transistors is biased for the worst-case damage to occur, and the off-transistors are just the opposite. This differential damage condition due to the asymmetrically applied bias imprints the cell by establishing a preferred state, as dose accumulates. One can write a memory with all "ones" stored in it during irradiation. Accumulated dose will make the "one" pattern a very hard pattern. If one now writes in all "zeros," that is, the complement pattern, then the error cross-section is now significantly increased. An experimental example of this is shown in Figure 10 (11), where the error cross-section versus LET is shown for a HARRIS $6504 \mathrm{RH}, 4 \mathrm{Kx} 1 \mathrm{CMOS} / \mathrm{Bulk}$ SRAM irradiated to a dose of 3 megarad. Half of the memory was measured for errors as a function of LET with the soft (or complementary) bits stored, and the same was done with the hardened (or preferred) bits stored. The curves for these two sectors and the curve for a control, that is, an unirradiated part, are shown in this figure. It can be seen that the threshold LET is lower for the soft sector compared to the other, and the asymptotic cross-section for the soft sector is greater than the control and the hard sector. Of course, the cross-section is lower for the hard part relative to the control.

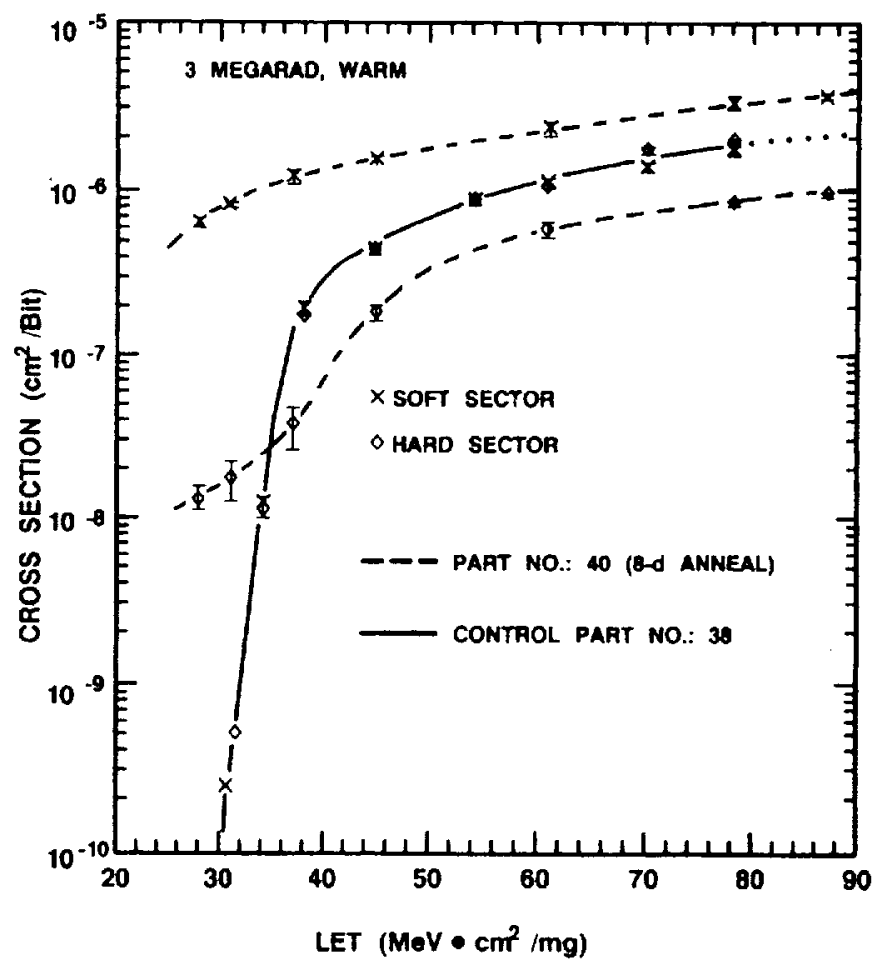

Figure 10. Average Cross-Sections vs. LET for 3-Megarad, Warm, Imprinted Memories. 


\section{F. Basic Upset Cross-Section Curves versus LET}

Error cross-section curves consist of three basic shapes. There is a classical shape which shows an abrupt and rapid increase in error rate with increasing LET similar to a square pulse waveform. Most parts show a slower rate of increase with a variety of slopes, but finally reaching a plateau or asymptotic level. Then there is a third type which resembles a staircase where the device contains many nodes collecting charge with a series of threshold LET values. The first type of curve provides a welldefined threshold LET and a saturated or asymptotic crosssection, thus, more accurate upset predictions may be calculated. The other two cases are more usual and prevalent in devices, and consequently, they have led to several definitions of threshold LET, depending on the individual researcher. Figure 11 illustrates these three cases. The staircase curve has been experimentally observed for a bipolar device. The bipolar technology contains a larger number of charge collection nodes than CMOS/ Bulk, and there are even fewer for CMOS/SOS or SOI devices. Thus, it can be seen that as the threshold LET is reached for any node, the cross-section increases, levels off, and then the next node takes over until finally the hardest node is reached and a final plateau in the cross-section is achieved.

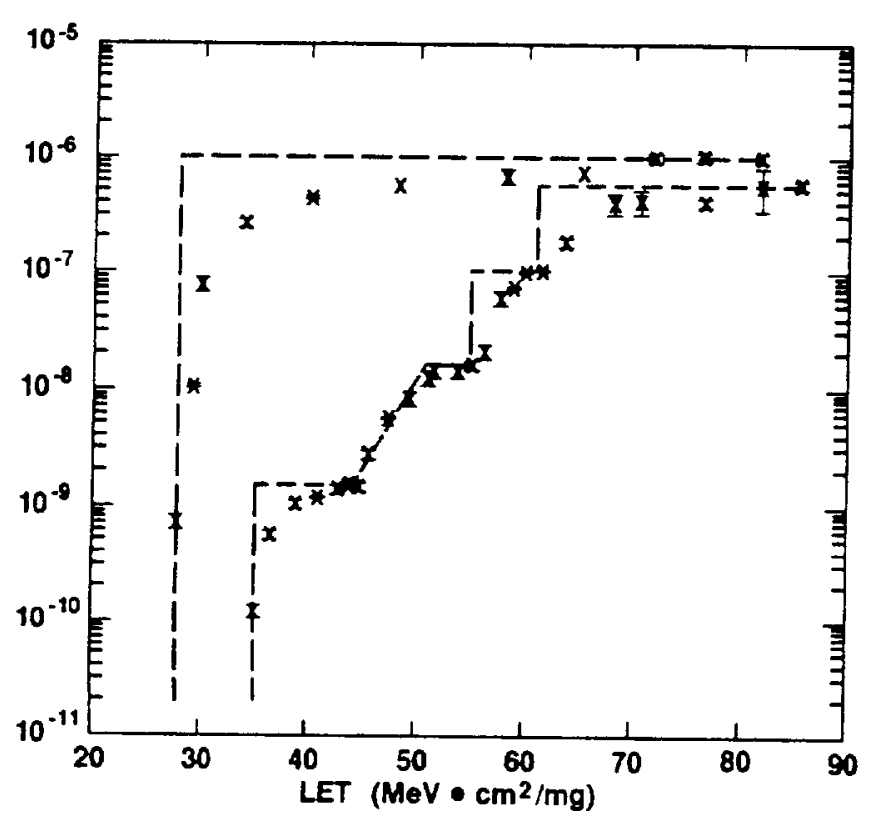

Figure 11. Three Typical Shapes of Upset Cross-Section Curves vs. LET.

\section{G. Arbitrary Definition of Threshold LET}

The previous section discussed the three basic shapes of cross-section curves versus LET. The non-classical shapes 2 and 3 have led to arbitrary definitions of threshold LET, since the threshold value is necessary to calculate and predict error rates of devices in a spacecraft system. Some of these, which have been postulated by various workers, are the values of LET at $10 \%$, $30 \%$, or $50 \%$ of the asymptotic cross-section, and also the LET when the first few upsets occur. This latter one is the lowest value, and, therefore, it will predict the highest or most conservative error rate. The correct definition to use is the one that produces the best agreement with space observations.

\section{$H$. Integration versus Non-Integration}

There is a way to avoid the arbitrary selection of threshold LET, and that is by utilizing the total cross-section versus LET curve if the experimental data is not al ways available. However, the more accurate approach is to integrate the error curve over the LET values and the environment. This procedure was applied to the data obtained with imprinted CMOS/Bulk 4Kxl HARRIS $6504 \mathrm{RH}$ parts, which was described in a previous section (SEU Concerns: Arbitrary Definition of Threshold LET). Figure 12 shows the upset rate in errors/bit-day versus the doses used in the imprinting experiments, for soft and hard sectors of these memory samples and for integrated and non-integrated calculations (13). It can be seen that the integrated approach yields error rates that are about a factor of 10 less than by the use of an arbitrary definition of LET. Of course, this will provide the best predictions; best agreement with space observations is still controlled by the accuracy of the environment used in the calculations (14, $15)$.

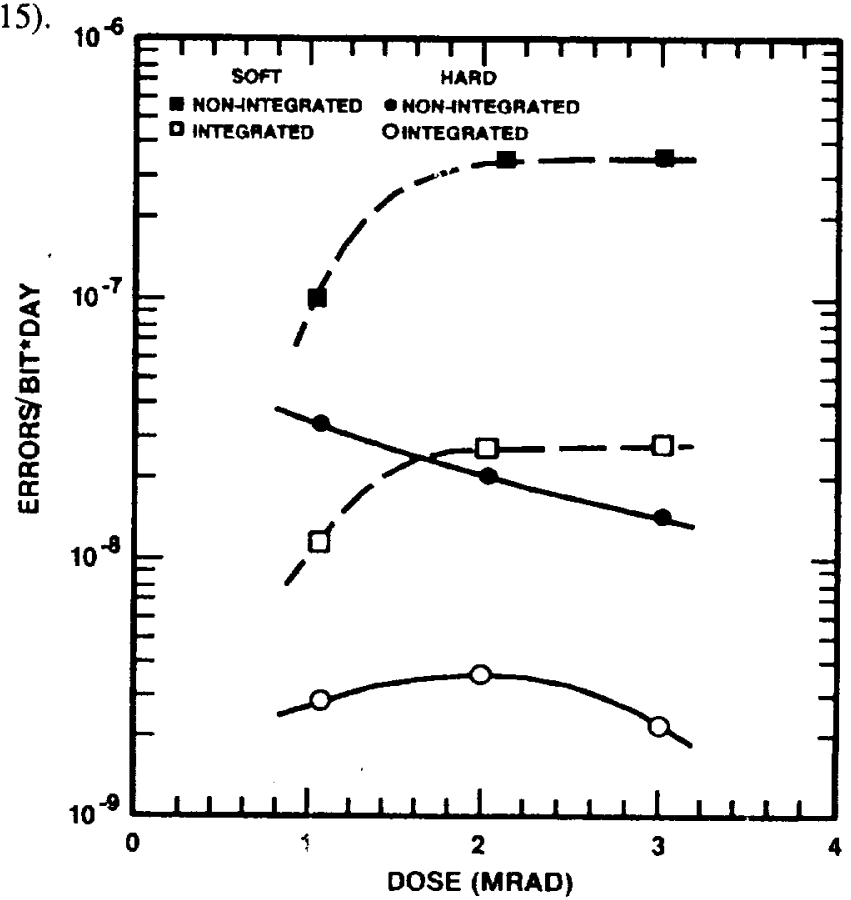

Figure 12. Unannealed Imprinted Memory Upsel Rates (Integrated and Non-Integrated vs. Ionization Dose.

In applying an upset code, one must input the dimensions of the sensitive volume. The cross-sectional area of this volume can be calculated directly from the asymptotic cross-section obtained by SEU testing. However, the depth "d" of this volume has heretofore been assumed or inferred from measurements. Recent work has indicated that this dimension can be determined from proton tests. Figure 13 illustrates that regardless of the value of depth selected, the ratio of non-integrated to the integrated results shown previously in Figure 12 is a constant and is independent of depth in the range of 1 to 7 micrometers (13). The other fact 
to note is that the smaller value of "d" will give the highest, or more conservative, error rate in either approach.

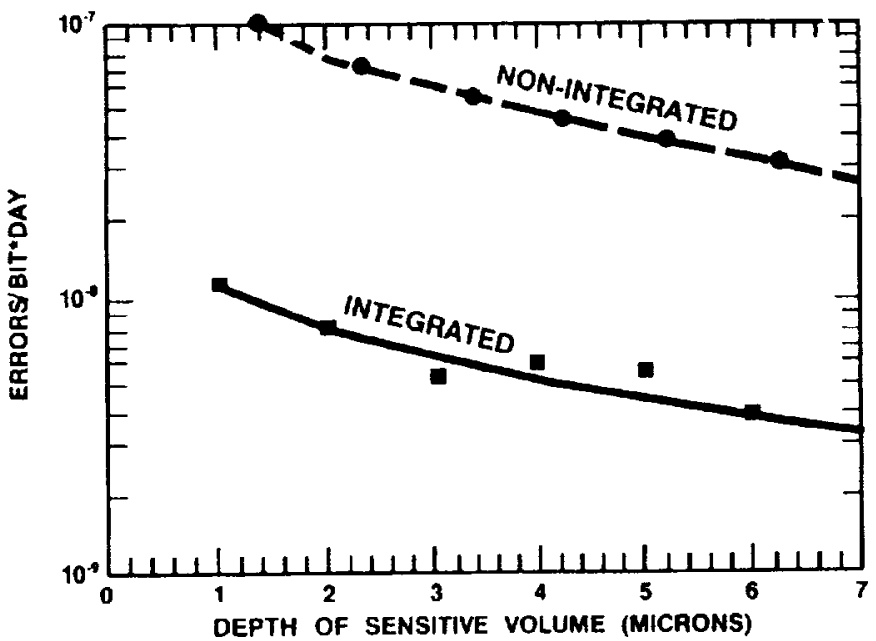

Figure 13. Comparison of Integrated to Non-Integrated Error Rates vs. Depth.

\section{Multiple Upsets}

A single ion hit can produce more than one error, that is, multiple errors can be created. Whether or not this happens is simply a function of the spacing of the device upset nodes which can share the ionization charge. The total charge created by the ion hit has to be equal to the sum of the critical upset charge for each node and it has to be collected by these nodes. The potential for multiple upsets to occur increases as the feature size decreases. Certainly 1.25-micrometer technology and small size devices, which are now available in the marketplace, makes this possibility very probable. In space, the availability of higher ion energies and all angles of incidence makes the occurrence of multiples very possible, and recently there have been reports of multiples observed in space $(16,17)$.

This has become an important issue since multiple errors in ground tests can contaminate data and may lead to error crosssections that are too high. In space, they produce higher upsets than predicted and if two errors occur in a single memory word, then these errors can defeat Error Detection and Correction (EDAC) hardware. Most of the EDAC devices will detect two errors in a word but will not correct them.

Data obtained from the "imprint experiments" $(11,18)$ (see section "SEU Concerns: B. Objectives and Changes in SEU Sensitivities"), show that multiples occurred mainly at high LETs and the majority of them were in the soft sectors; that is, in the sectors where the upset cross-section was highest. The results indicated that singlets, doublets, and triplets were produced by single ion hits, and the number of each was a function of LET, ionization dose, and cross-section (for soft and hard sectors). A closer definition of a "multiple hit" does not exist at this time, but multiple upsets can be arbitrarily defined to be two or more physically adjacent errors that are assumed to be produced by a single ion hit. Workers in this field have used this approach (17, $18,19,20,21,22,23)$. Experimentally, if one could achieve a single ion exposure of a device and count the number of errors after the hit, then the ambiguity would be climinated. These errors could only be multiples, regardless of their location in the memory.

In recent work, a doublet was arbitrarily selected to be any pair of upsets in two physically adjacent locations, with combinations in any direction. In the case of a triplet, it was defined in a similar manner, including all permutations. Figure 14 shows the number of total events for a group of imprinted samples irradiated to some dose versus total number of upsets for each ion run (18). The "total" means that the sum of errors in both soft and hard sectors was plotted. This is the data for the parts in the 1megarad group. Predictions based on Poisson statistics are shown together with the experimental data for singlets, doublets, and triplets. The agreement between theory and experiment is good at this dose level. Disagreement increased for the 2- and 3megarad groups of samples. One explanation is that the theoretical predictions require random events whereas the randomness of the errors decreases as the dose or imprinting increases.

As stated above, the ideal experiment is to use only a single ion per exposure. A recent experiment, which was a step in this direction, utilized an improved technique which basically reduced the resolution time for memory readout of data from one minute to one second. This technique essentially increased by one order of magnitude the possibility that multiple ions rather than a single ion caused the apparent multiple upsets, reported in (18). Now, these new results indicate that the occurrence of a doublet or triplet, as shown in Figure 14, is very improbable. These recent results should modify the community's thinking relative to the possibility of multiple errors, not only for the particular part type tested in this work, but also for the probability of multiples occurring in a general sense.

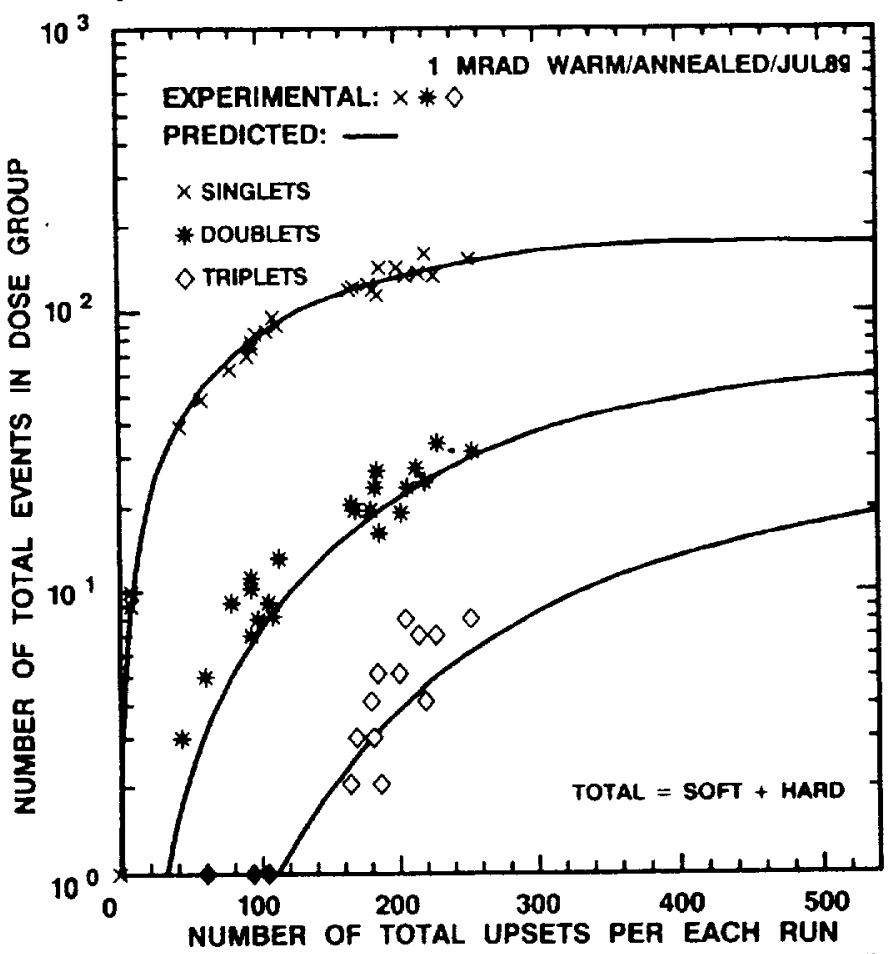

Figure 14. Comparison of Total Multiple Upsets (= Soft + Hard) vs. Events, for Imprinted Memories. 


\section{J. Burnout}

A single ion hit can cause burnout of a power MOSFET. There has been a major effort to characterize this effect since that is a very popular device for use in a power converter or for switching high currents in spacecraft systems. In particular, MOSFETs are used extensively in communication satellites in geostationary orbits. The generation of a density of electron-hole pairs in the sensitive region of the MOSFET produces a flow of current that forward-biases the parasitic bipolar transistor.

This transistor, which is formed by the n-source, $\mathrm{p}$-body, and $\mathrm{n}$-drain draws an ever-increasing current; subsequently, second breakdown occurs and thermal meltdown of the aluminum gate, with it alloying to the silicon. The destruction is catastrophic and the device fails. Ground tests have accumulated a large amount of data for many types of MOSFETs. The results indicate that burnout takes place when the drain-source voltage is approximately equal to $50 \%$ of the breakdown voltage. This simple rule of thumb is only good to about 20 volts and depends significantly on the ion's LET. Up to this time, there have been no reports of MOSFET burnout in space. This has been a mystery as to why, since there are power MOSFETs (the IRF 150) flying in an RCA communications satellite in a geostationary orbit without burnout after more than 165,000 device-days. A very recent test may have provided the answer to this survival mystery (24). Figure 15 shows a plot of non-destructive bumout cross-sections versus LET for 65-volt drain-source voltages taken in a static mode, whereas Figure 16 is a plot of destructive burnout that was obtained operating the parts dynamically by applying a $63-\mathrm{kHz}$ square wave to the gates of the devices for 65 -volt drain-source voltages. Comparison of this data shows that the dynamic operation reduced the cross-section by about a factor of 100 compared to the static case. This difference appears to be the answer to the unusual survivability of the devices in space, since they are operated dynamically at a frequency of $50 \mathrm{kHz}$, as in the ground experiments.

The actual absolute difference in cross-section is governed by Poisson statistics, thus sample size is important. The plot in Figure 16 was obtained by dividing each event by the fluence at which the event took place, and these are the values shown in Figure 16. Consideration of Poisson statistics suggests that a better approach is to sum all of the events for all samples per LET and drain-source voltage, and then to divide this value by the sum of all sample fluences. This approach increases the static to dynamic cross-section ratio from 100 to 1000 (ref. 25).

\section{K. Latchup in CMOS/Bulk Devices}

An ion hit in the sensitive volume of a CMOS/Bulk part can initiate a typical SCR (Silicon Controlled Rectifier) pnpn latchupwhich may lead to burnout of the device. It is the npn and pnp parasitic transistors that make this effect possible. Also, this effect is more probable as the feature size of parts decreases. For example, unless special processing is used, $1.25-\mu \mathrm{m}$ feature size parts have a very high cross-section and low threshold LET for latching to occur. The hardening approach to avoid latchup in a small feature size part is to fabricate the device on epitaxial

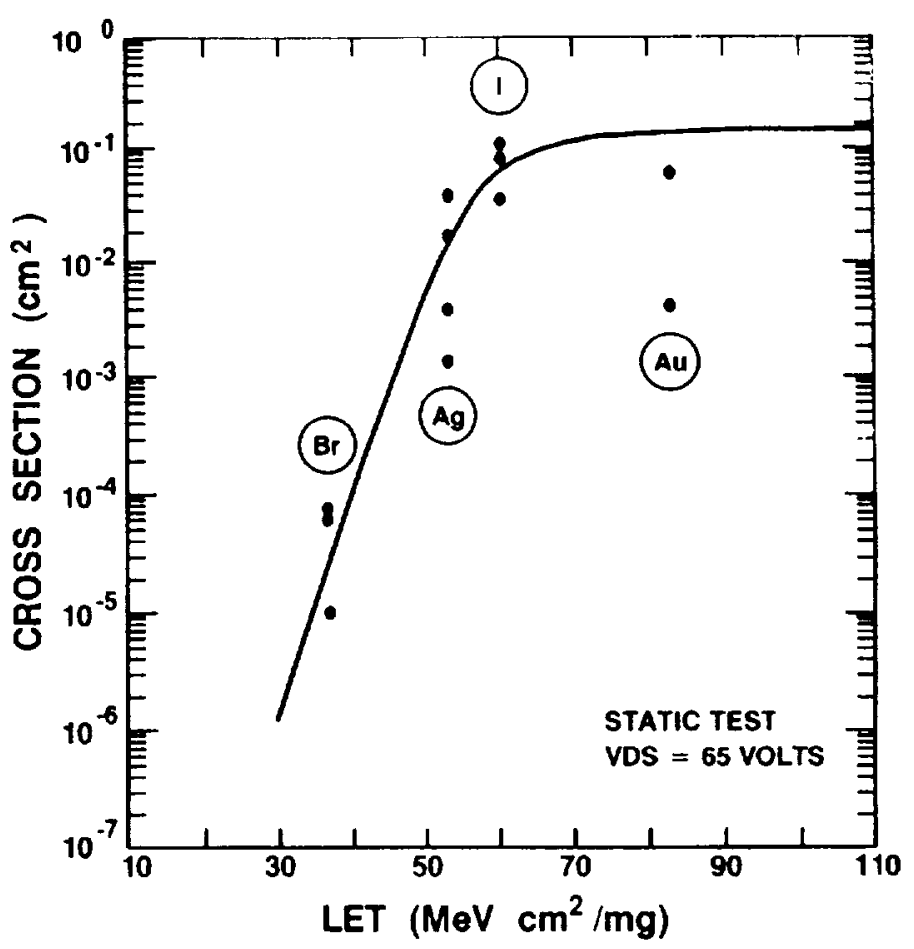

Figure 15. Non-Destructive Burnout Cross-Sections of Power MOSFETs vs. LET for Static Test Results ( $V_{D s}=65$ Volts).

\section{NON-DESTRUCTIVE SEB CROSS SECTIONS VS. LET}

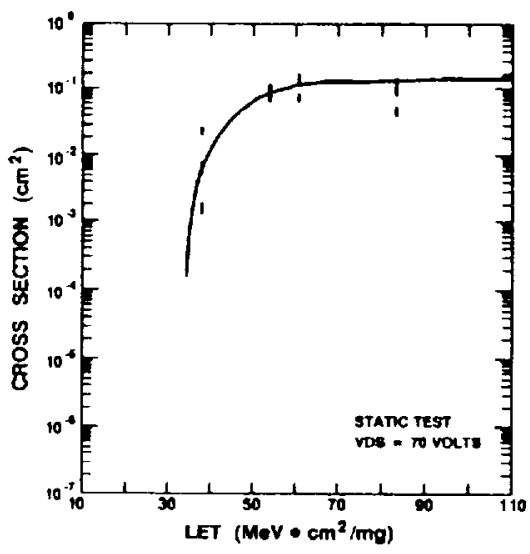

DESTRUCTIVE SEB CROSS SECTIONS VS. LET

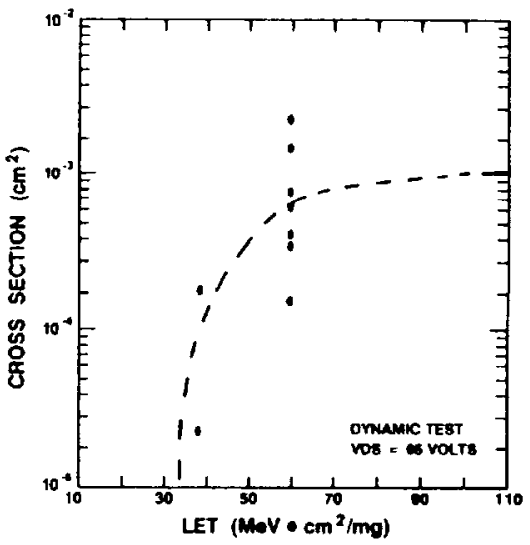

Figure 16. Non-Destructive and Destructive Burnout Cross Sections of Power MOSFETS vs. LET for Dynamic Test Results $\left(V_{D s}=65\right.$ Volts $)$. 
silicon or to use dielectric isolation. Thus, CMOS/SOS and CMOS/SOI are immune to latchup. Clearly, devices that may latch could be ground tested and eliminated from spacecraft subsystems if they prove to be latchup prone. High temperatures increase the cross-section for latching. Therefore, if the application in space requires elevated temperatures of operation, then the ground test must be carried out with the devices at these temperatures.

\section{Transient Upset (Glitches) in Fiber Optics}

Heavy ions generate ionization charge in any silicon device. In simple logic gates that do not contain storage elements--for example, in memories, shift registers, counters, and flip-flops-this charge will produce a current and a subsequent glitch. This voltage signal may propagate to a latch somewhere in the system design where it may trigger the latch. This change of state can produce an upset or error. In general, workers have not tested non-storage devices or searched for glitches. It is mainly an issue of a system. For example, a computer made up of I/O devices, glue chips, and a microprocessor is a potential system where glitches generated in the $\mathrm{I} / \mathrm{O}$ devices may be propagated to registers in the microprocessor and cause problems. A recent investigation (26) of a fiber optic communication system for the occurrence of glitches showed that they could be generated in this system, and thus, bit errors were produced, contaminating the transmitted and received data. Figure 17 is a plot of the upset cross-section for the receiver and transmitter of a fiber optic system versus LET. The transmitter and receiver were irradiated separately. The curves are for the dynamic operating mode and the results show that the receiver is the worst case, with a very high asymptotic cross-section of $.07 \mathrm{~cm}^{2}$. The transmitter has a cross-section of about $3 \times 10^{-4} \mathrm{~cm}^{2}$ and is less likely to experience a glitch-induced upset than is the receiver.

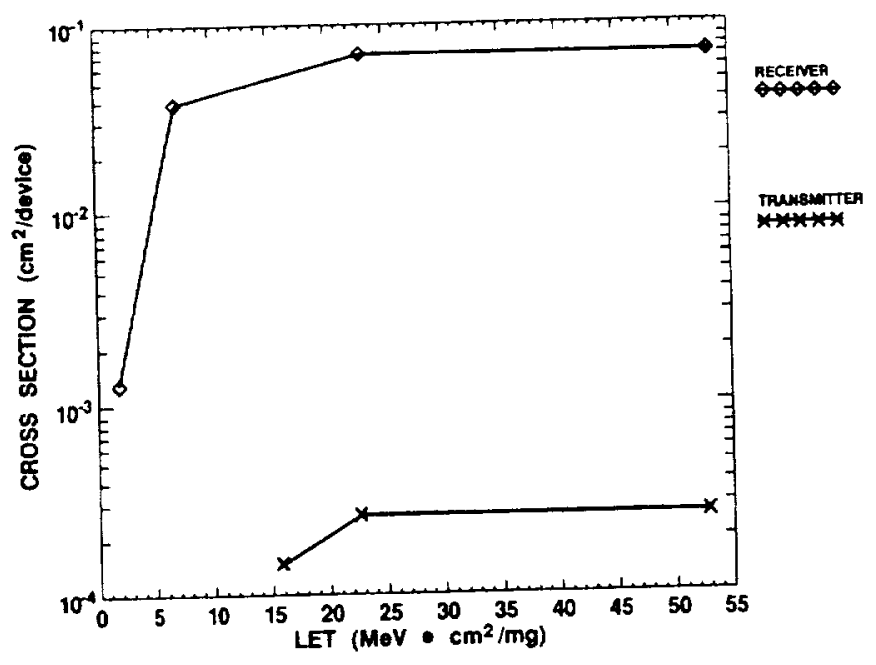

Figure 17. Filtered Average Glitch Cross-Sections of RECB and TX for Dynamic Case (100-kHz Square Wave).
Figure 18 shows the corresponding data taken with protons at three energies. Again, there is a difference in sensitivity of about two orders of magnitude for the receiver compared to the transmitter. Note the sharp drop-off in cross-sections as the proton energy was increased from $148 \mathrm{MeV}$ to about $160 \mathrm{MeV}$. There is no explanation at this time for this sudden decrease. Itappears to be valid data since it occurred in two samples of the receiver and also in two samples of the transmitter in two separate experiments. In further support of the data validity is the apparent threshold at $150 \mathrm{MeV}$ for both devices.

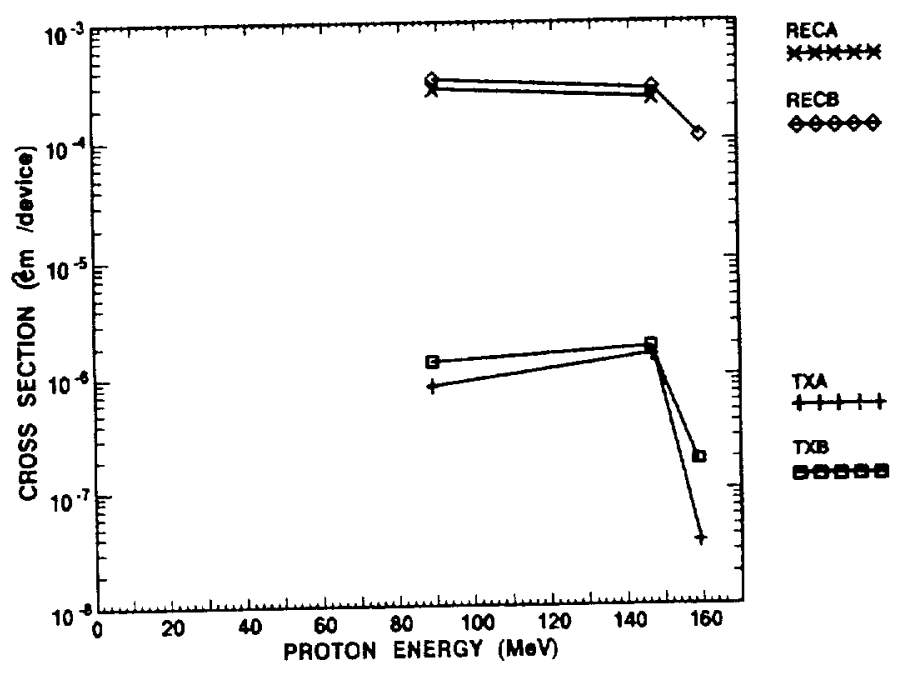

Figure 18. Average Glitch Cross-Sections of Receivers and Transmitters for Dynamic Tests vs. Proton Energies.

\section{Proton Upsets and Concerns}

Protons can induce upsets in devices by interaction with $\mathrm{Si}$ atoms, which in turn, generate secondary particles. It is these secondaries that produce the ionization charge that causes upsets. Most parts with relatively high LET thresholds (e.g., higher than LET $=20 \mathrm{MeV}-\mathrm{cm}^{2} / \mathrm{mg}$ ) will not be upset by protons. The limiting value seems to be about LET $=15 \mathrm{MeV}-\mathrm{cm}^{2} / \mathrm{mg}$ ) for a device to be upset by high-energy protons. Protons can also cause glitches in sensors (e.g., star trackers) and interfere with the normal operation of such instruments. Bipolar devices that have particularly low threshold LET values (e.g., $1-5 \mathrm{MeV}-\mathrm{cm}^{2} / \mathrm{mg}$ ) for heavy ions will upset at a high rate in regions of space with large proton populations.

Predictions of proton upsets in space require that upset parameters be obtained. This is usually accomplished by measuring the upset cross-section as a function of proton energy. The 
MK4116J-2

UPSET CROSS SECTION (cm $2 / \mathrm{bit})$

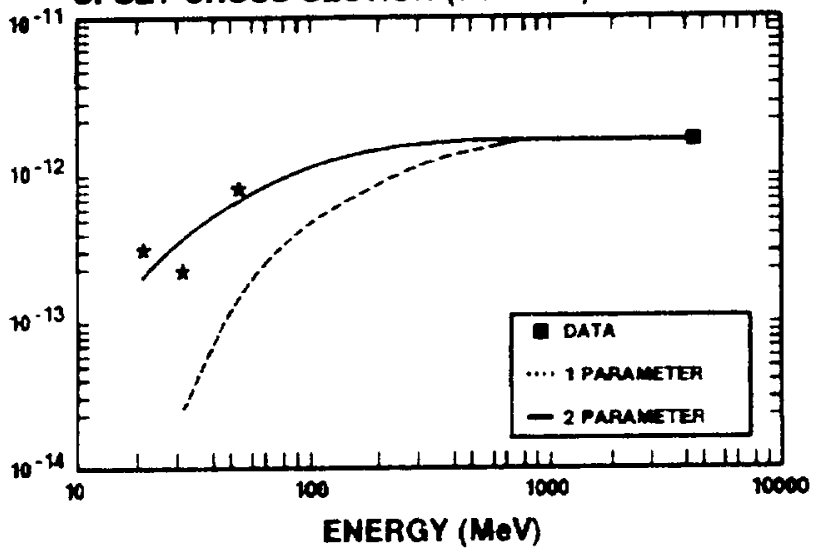

$93 L 422$

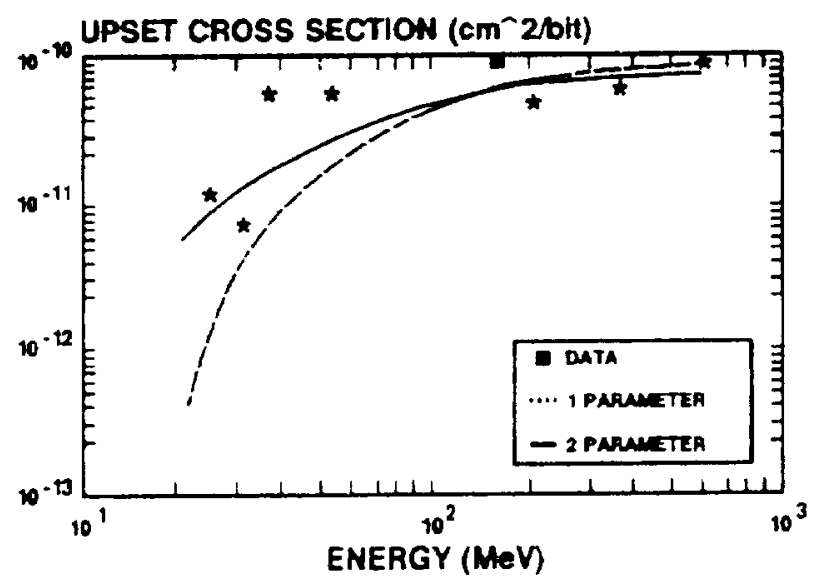

$8 \times 350$

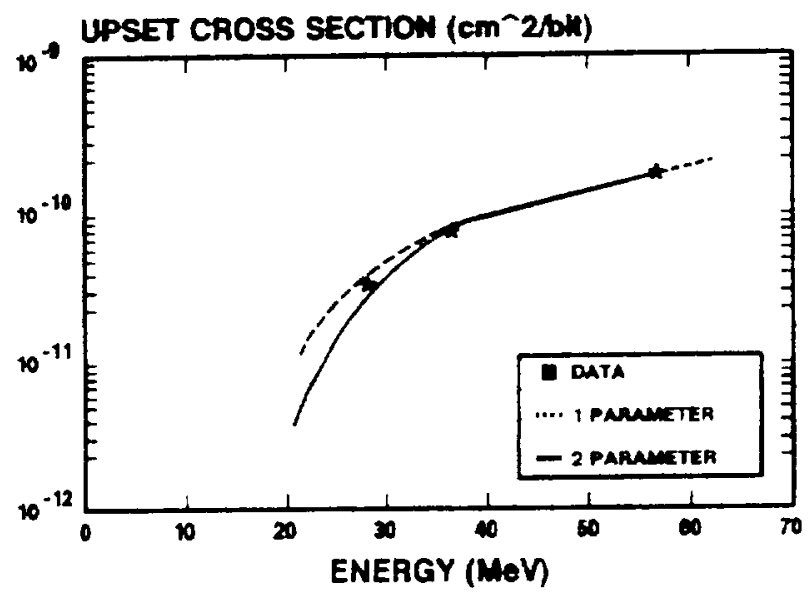

93425

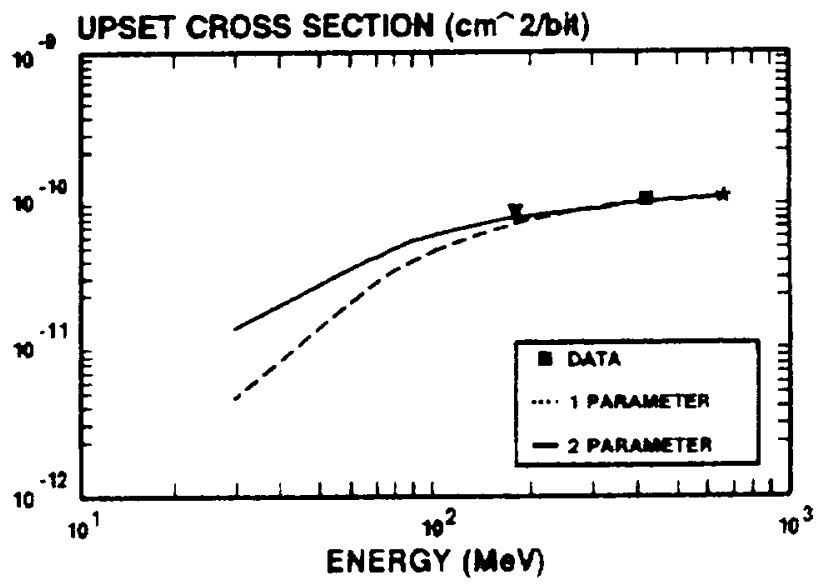

Figure 19. Upset Cross Sections vs. Proton Energy for several different part types.

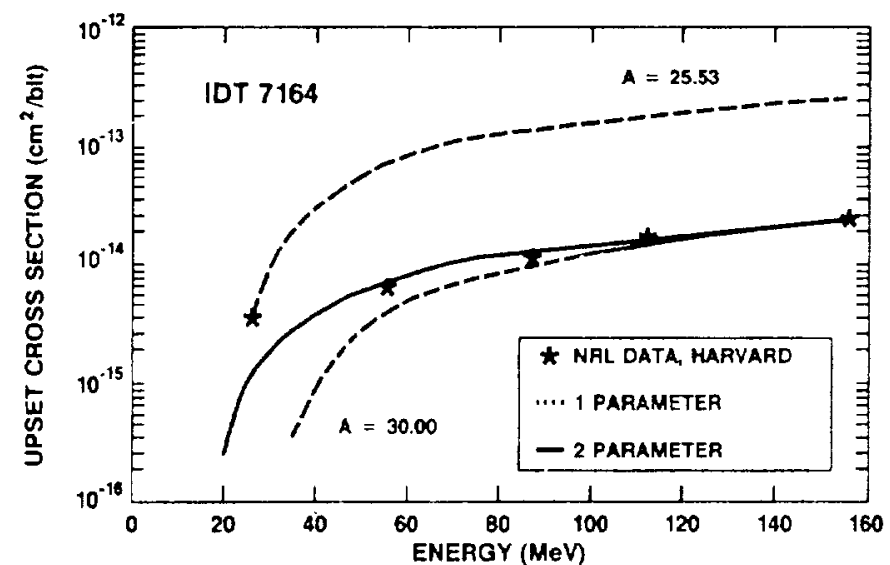

Figure 20. Upset Cross Sections v. Proton Energy for an IDT 7164 device. resulting curve will allow a determination of the Bendel parameter, which is needed for the calculation of upset rates. Present data have shown that for some devices a better fit to this experimental curve can be made by using a 2-parameter approximation rather than the 10 -parameter approach. Figures 19 and 20 show proton cross-sections versus proton energy for a number of parts. For the part types shown in these figures, the 2-parameter approach appears to do a better fitting job than the Bendel 1parameter method (27). The 1-parameter algorithm historically has been used to predict proton upsets in space.

\section{Conclusions and Recommendations}

It is recognized from the discussion of the many shortcomings and concerns presented in this paper that ground tests are, at best, a compromise based on limits of time, facilities availability and capabilities, and cost considerations. 
The same holds true for the methods and procedures used in performing experiments, executing diagnostics, employing dosimetry, and performing calibrations. The uncertainties in any or all of these may be significant.

In addition, the inadequacies of the units of radiation and stopping power (LET) to account for induced effects and the unaccounted-for inequality of radiation types and energies in producing effects, may contribute to the overall inaccuracies.

It has also been shown that in laboratory lests, the level of ionizing dose, the rate of exposure, and the time of measurement play important roles in correctly assessing damage in electronic parts or systems.

During irradiation experiments and SEP testing, parameters such as temperature, bias, and mode of operation may substantially affect the results obtained and hence, the conclusions drawn.

Annealing, recovery, or rebound may play an important role in the lifetime of a given part, but in most cases, accelerated testing and time constraints do not allow a complete and thorough definition of their impact on the long-term performance of the part.

Calculations are another source of uncertainty, errors, and inaccuracies, particularly in the areas of shielding and transport, LET threshold definition, evaluation of effects (integration vs. non-integration), proton upset predictions using a 1- or 2-parameter approach, critical charge estimation, and physical dimension approximations of sensitive volume (when not provided by vendor), etc.

Similarly, imprint effects, multiple errors due to single particles, and impact of operational mode (dynamic versus static) are some other areas of concern that may affect the use, application, and interpretation of results.

Within these general or specific constraints then, the following are some recommendations that may be feasible and practical so as to improve the overall prediction capability, and reliability for electronic parts and systems in the space radiation environment:

1. Establish a better approximation to the space environment by irradiating the silicon die with electron and proton sources of different energies, and not with Co-60 gamma rays alone, which do not exist in space.

2. Improve evaluation of radiation effects and prediction levels by an accurate shielding analysis of a spacecraft and all its subsystems, including the device packaging.

3. Determine the contribution of low-energy electrons $(\leq$ $100 \mathrm{KeV}$ ) and protons ( $\leq 5 \mathrm{MeV}$ ) to the total ionization dose of the spectra hitting the die. If significant, modify Cobalt- 60 test dose for accepting parts.

4. Account for PIE effects in Cobalt-60 tests of MOS parts by using an accelerated, high dose-rate exposure combined with some high-temperature annealing procedure and subsequent long-term measurements of electrical parameters.
5. Design Cobalt-60 test setups to simulate (a) device application in a space subsystem, at least as to whether dynamic or static operation is appropriate, and (b) operating temperature in space.

6. Select facility and ion types for SEU testing that satisfy stability, beam purity, ease of testing, range requirements, etc., of parts to be tested, and use an ion only at normal incidence, unless the application of $\cos \theta$ to normalize LET is known to be a valid concept.

\section{Test for SEU and SEL at space operational temperatures.}

8. Test for dose imprint effect if dose environment of application is significant.

9. Check for SEU transient generation as well as other effects.

10. Design the SEU test setup to detect and measure multiple errors, or design an experiment to minimize them.

11. Whenever device data include $\sigma$ vs. LET curves, use integration of $\sigma$ over LET curve.

12. For devices designated for use in an environment containing high proton fluences and for which threshold LET is $\leq 20$, conduct tests with protons.

13. Power MOSFET tests for heavy-ion-induced bumout should be carried out at the operating frequency if application is a dynamic one.

All of the shortcomings and effects described here also impact the issue of spacecraft design margins. Clearly, space predictions of electronic systems survival from ionization damage or heavy-ion effects are dominated by test data. In turn, overdesign or underdesign are undesirable results of inaccurate predictions. Overdesign margins lead to excess weight and unwanted launch costs, and of course, underdesign margins can shorten mission life. 


\section{References}

[1] McNulty, P., private communication, 1991.

[2] Stassinopoulos. E.G. and James P. Raymond, "The Space Radiation Environment for Electronics," IEEE Proceedings, Vol. 76, No. 11, November 1988.

[3] Brucker, G.J., E.G. Stassinopoulos, O. Van Gunten, L.S. August, and T.M. Jordan, "The Damage Equivalence of Electrons, Protons, and Gamma Rays in MOS Devices," IEEE Trans. Nucl. Sci., Vol. NS-29, No. 6, December 1982.

[4] Stassinopoulos, E.G., G.J. Brucker, O. Van Gunten, A.R. Knudson, and T.M. Jordan, "Radiation Effects on MOS Devices: Dosimetry, Annealing, Irradiation Sequence, and Sources," IEEE Trans. on Nucl. Sci., Vol. NS-30, No. 3, June 1983.

[5] Brucker, G.J., O. Van Gunten, E.G. Stassinopoulos, P. Shapiro, L.S August, and T.M. Jordan, "Recovery of Damage in Rad-Hard MOS Devices During and After Irradiation by Electrons, Protons, Alphas, and Gamma Rays," IEEETrans. on Nucl.Sci., Vol. NS-30, No. 6, December 1983.

[6] Stassinopoulos, E.G., O. Van Gunten, G.J. Brucker, A.R. Knudson, and T.M. Jordan, "The Damage Equivalence of Electrons, Protons, Alphas and Gamma Rays in Rad-Hard MOS Devices," IEEE Trans. Nucl. Sci., Vol. NS-30, No. 6, December 1983.

[7] Brucker, G.J., "Exposure Dose Rate Dependence for a CMOS/SOS Memory," IEEE Trans. Nucl.Sci., Vol. NS-28, No. 6, December 1981.

[8] Stassinopoulos, E.G., G.J. Brucker, and O. Van Gunten, "TotalDose and Dose-Rate Dependence of Proton Damage in MOS Devices During and After Irradiation," IEEE Trans. Nucl. Sci., Vol. NS-31, No. 6, December 1984.

[9] Petersen, E.L., P. Shapiro, J.H. Adams, Jr., and E.A. Burke, "Calculations of Cosmic Ray Induced Soft Upsets and Scaling in VLSI Devices," IEEE Trans. Nucl. Sci., Vol. NS-29, No. 6, December 1982.

[10] Griswell, T.L., D.L. Oberg, J.S. West, P.R. Measel, and W.E. Wilson, "Measurements of SEU Thresholds and Cross Sections at Fixed Angles," IEEE Trans. Nucl. Sci., Vol. NS-34, No. 6, December 1987.

[11] Stassinupoulos, E.G., G.J. Brucker, O. Van Gunten, and H.S. Kim, "Variations in SEU Sensitivity of Dose-Imprinted CMOS SRAMs," IEEE Trans. Nucl. Sci., Vol. NS-36, No. 6, December 1989.

[12] Stapor, W.J., P.T. McDonald, A.B. Campbell, A.R. Knudson, and B.G. Glagola, "Charge Collection in Silicon for Ions of Different Energy but Same Linear Energy Transfer (LET)," IEEE Trans. Nucl. Sci., Vol. NS-35, No. 6, December 1988.

[13] Brucker, G.J. and E.G. Stassinopoulos, "Prediction of Error Rates in Dose-Imprinted Memories On Board CRRES by Two Different Methods," IEEE Trans. Nucl. Sci., Vol. NS-389, No. 3, June 1991.

[14] Shoga, M., P. Adams, D.L. Chenette, R. Koga, and E.T. Smith, "Verification of Single Event Upset Rate Estimation Method With OnOrbit Observations," IEEE Trans. Nucl. Sci., Vol. NS-34, No. 6 , December 1987
[15] Harboe-Sorensen, R.L., L. Adams, E.J. Daly, C. Soursoe, D. Mapper, and T.K. Sanderson, "The SEU Risk Assessment of Z80A, 8086, and 80C86 Microprocessors Intended for use in a low-Altitude Polar Orbit," IEEE Trans. Nucl. Sci., Vol. NS-33, No. 6, December 1986.

[16] Campbell, A., "SEU Flight Data from the CRRES MEP," to be published in IEEE Trans. Nucl. Sci., Vol.NS-38, No. 6, December 1991.

[17] Blake, J.B. and R. Mandel, "On Orbit Observations of Single Event Upset in Harris HM-6508 1K RAMS," IEEE Trans. Nucl. Sci., Vol. NS33, No. 6, December 1986.

[18] Stassinopoulos, E.G. and G.J. Brucker, "Multiple Upset in Memories and Their Impact on Error Predictions and Subsystem Designs," to be presented at the RADECS Conference, September 1991. To be published in IEEE Special Proceedings for RADECS, 1991

[19] Zoutendyk, J.A., L.D. Edmonds and L.S. Smith, "Characterization of Multiple Bit Errors from Single Ion Tracks in Integrated Circuits," IEEE Trans. Nucl.Sci., Vol. NS-30, No. 6, December 1989

[20] Zoutendyk, J.A., L.S. Smith, and L.D. Edmonds, "Response of a DRAM to Single Ion Tracks of Different Heavy Ion Species and Stopping Powers," IEEE Trans. Nucl. Sci., Vol. NS-37, No. 6, December 1990 .

[21] Koga, R., W.R. Crain, K.B. Crawford, D.D. Lau, S.D. Pinkerton, K.K. Yi, and R. Chitty, "On the Suitability of Non-Hardened High Density SRAMs for Space Applications," to be published in IEEE Trans. Nucl. Sci., Vol. NS-38, No. 6, December 1991

[22] Koga, R., W.A. Kolasinski, J.V. Osborn, J.H. Elder, and R. Chitty, "SEU Test Techniques for $256 \mathrm{~K}$ Static RAMs and Comparisons of Upsets by Heavy Ions and Protons," IEEE Trans. Nucl. Sci., Vol. NS-35. No. 6, December 1988.

[23] Song, Y., K.N. Vu, J.S. Cable, A.A. Witteles, W.A. Kolasinski, R. Koga, J.H. Elder, J.V. Osborn, R.C. Marlin, and N.M. Ghoniem, "Experimental and Analytical Investigation of Single Event, Multiple Bit Upsets in Poly-Silicon Load, 64Kx1 NMOS SRAMs," IEEE Trans.

Nucl. Sci., Vol. NS-35. No. 6, December 1988.

[24] Calvel, P., E.G. Stassinopoulos, C. Peyrotte, and A. Baiget, "Comparison of Experimental Measurements of Power-MOSFET SEBs in Dynamic and Static Modes," to be published in IEEE Trans. Nucl. Sci., Vol. NS-38, No. 6, December 1991.

[25] Stassinopoulos, E.G., G.J. Brucker, and P. Calvel, "Charge Generation by Heavy Ions in Power MOSFETs, Burnout Space Predictions, and Dynamic SEB Sensitivity," submitted to IEEE-NSREC, New Orleans, LA, July 1992.

[26] LaBel, K. and E.G. Stassinopoulos, "Transient SEUs in a Fiber Optic System for Space Applications," to be published in IEEE Trans. Nucl. Sci., Vol. NS-38, No. 6, December 1991.

[27] Stapor, W.J., J.P. Meyers, J.B. Langworthy, and E.L. Petersen, "Two Parameter Bendel Model Calculations for Predicting Prown Induced Upset," IEEE Trans. Nucl. Sci., Vol. NS-37, No. 6, December 1990. 




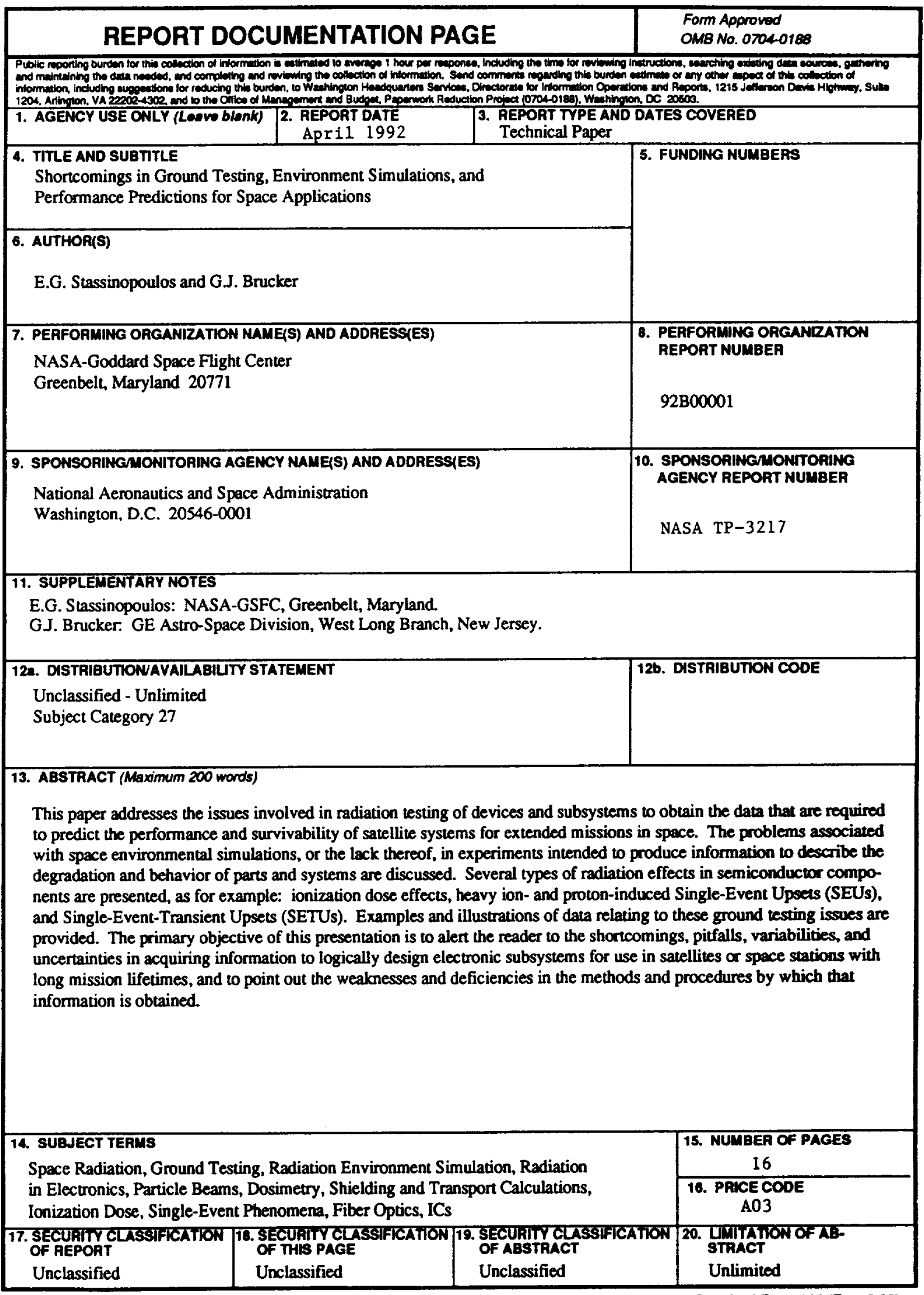

